\title{
Discrete and Lexicographic Helly-Type Theorems
}

\author{
Nir Halman
}

Received: 26 January 2006 / Revised: 15 April 2007 /

Published online: 18 September 2007

(C) Springer Science+Business Media, LLC 2007

\begin{abstract}
Helly's theorem says that if every $d+1$ elements of a given finite set of convex objects in $\mathbb{R}^{d}$ have a common point, then there is a point common to all of the objects in the set. We define three new types of Helly theorems: discrete Helly theorems-where the common point should belong to an a-priori given set, lexicographic Helly theorems-where the common point should not be lexicographically greater than a given point, and lexicographic-discrete Helly theorems. We study the relations between the different types of the Helly theorems. We obtain several new discrete and lexicographic Helly numbers.
\end{abstract}

Keywords Helly theorems · Discrete geometry

\section{Introduction}

The classical theorem of Helly stands at the origin of what is known today as the combinatorial geometry of convex sets. It was discovered in 1913 and may be formulated as follows.

Theorem 1.1 (Helly's Theorem) Let $H$ be a family of closed convex sets in $\mathbb{R}^{d}$, and suppose either $H$ is finite or at least one member of $H$ is compact. If every $d+1$ or fewer members of $H$ have a common point, then there is a point common to all members of $H$.

An extended abstract containing parts of this work appeared in the proceedings of the Forty-Fifth Annual Symposium on Foundations of Computer Science (FOCS) 2004.

This work is part of the author's Ph.D. thesis, prepared in the school of mathematical sciences at Tel Aviv University, under the supervision of Professor Arie Tamir.

N. Halman ( $\square)$

Massachusetts Institute of Technology, Cambridge, MA 02139, USA

e-mail: halman@mit.edu 
A possible generalization of Helly's Theorem is as follows. Let $H$ be a family of objects, and let $\mathcal{P}$ be a predicate on subsets of $H$. A Helly-type theorem for $H$ is of the form:

There is a constant $h$ such that for every finite subset $G \subseteq H, \mathcal{P}(G)$, if and only if, for every $F \subseteq G$ with $|F| \leq h, \mathcal{P}(F)$.

The minimal such constant $h$ is called the Helly number of $H$ with respect to the predicate $\mathcal{P}$. If no such constant exists we say that the Helly number of $H$ with respect to $\mathcal{P}$ is unbounded or infinite $(\infty)$. In Helly's Theorem, the Helly number is $d+1$ and $\mathcal{P}$ is the predicate of having a non-empty intersection. In order to distinguish between these Helly-type theorems and the ones we define below, we call the former standard (or continuous) Helly theorems.

Over the years, a vast body of applications analogues and far reaching generalizations of Helly's Theorem has been assembled in the literature (see for instance the excellent surveys of $[4,6,8])$.

We present in this paper three ways to generalize Helly-type theorems. For instance, considering Helly's Theorem, we are interested in the least integer $h_{L}(d)$ such that the following lexicographic Helly's Theorem holds. (Recall that for every $x=\left(x_{1}, \ldots, x_{d}\right), y=\left(y_{1}, \ldots, y_{d}\right) \in \mathbb{R}^{d}, x$ is said to be lexicographically smaller than $y$ (lsmaller, in short, or $x<_{L} y$ ) if either $x_{1}<y_{1}$, or there exists $d \geq k>1$ such that $x_{i}=y_{i}$ for $i=1,2, \ldots, k-1$, and $x_{k}<y_{k}$.)

Lexicographic Helly's Theorem Let $H$ be a family of closed convex sets in $\mathbb{R}^{d}$ and let $x$ be a vector in $\mathbb{R}^{d}$. If every $h_{L}(d)$ or fewer members of $H$ have a common point which is lexicographically at most $x$, then all the members of $H$ do.

We call such $h_{L}(d)$ the lexicographic Helly number (lex Helly number, in short) of $H$.

We are also interested in the least number $h^{*}(d)$ such that the following discrete Helly's Theorem holds.

Discrete Helly's Theorem Let $D$ be a family of closed convex sets in $\mathbb{R}^{d}$ and let $S$ be a set of points in $\mathbb{R}^{d}$. If every $h^{*}(d)$ or fewer members of $D$ have a common point in $S$, then all the members of $D$ do.

We call such $h^{*}(d)$ the discrete Helly number of $H$. We consider combined lexicographic-discrete Helly-type theorems as well, that is, lexicographic-discrete Helly numbers (lex-discrete Helly numbers, in short) which we denote by $h_{L}^{*}$.

We note in passing that the lex Helly number of Helly's theorem applied to finite families of convex sets remains $h_{L}(d)=d+1$ (see for instance [19]), while when it is applied to infinite families of compact convex sets the lex Helly number becomes infinite (Example 2.3). We show in Sect. 2.1 that the discrete Helly number of Helly's theorem is infinite, and consequently by Observation 3.3 its lex-discrete Helly number is infinite as well. By restricting the shapes of the convex sets in $H$ we can get finite Helly numbers for the discrete and lex-discrete versions of Helly's Theorem (Theorem 2.10). 
Table 1 Continuous, discrete and lexicographic Helly numbers for 7 different cases of (finite) families of objects and properties

\begin{tabular}{|c|c|c|c|}
\hline Objects and property / type of Helly number & Continuous & Discrete & Lexicographic \\
\hline Non-empty intersection of convex sets in $\mathbb{R}^{d}$ & $d+1[17]$ & $\infty$ (Sect. 2.1) & $d+1[19]$ \\
\hline \multicolumn{4}{|l|}{ Non-empty intersection of axis-parallel } \\
\hline boxes in $\mathbb{R}^{d}$ & $2[3]$ & $2 d($ Thm. 2.10(i)) & $\max (2, d)($ Thm. 2.7(i)) \\
\hline 2-piercing of axis-parallel rectangles & $5[3]$ & $\infty($ Thm. 2.10(iii)) & 6 (Thm. 2.7(iii)) \\
\hline \multicolumn{4}{|l|}{ Ordered line transversal of an ordered family } \\
\hline of pairwise disjoint planar convex sets & $4[22,23]$ & 4 (Thm. 2.18) & 4 (Thm. 2.18) \\
\hline Line transversal of an ordered family of & $3[23]$ & 40 & 30 \\
\hline Line transversal of axis-parallel rectangles & $\begin{array}{l}3[25] \\
6[20]\end{array}$ & 8 (Thm. 2.12) & 6 (Thm. 2.12) \\
\hline Line transversal of pairwise disjoint & $5[10]$ & $7(\mathrm{Thm} 215(\mathrm{ii})$ & $5(\mathrm{Thm} 215(\mathrm{iii})$ \\
\hline
\end{tabular}

Our Results We obtain more than a dozen new such discrete, lexicographic and lex-discrete Helly numbers. We also study the relations between these new Helly numbers and their corresponding (continuous) Helly numbers. We summarize a part of our results in Table 1.

Applications Beyond the intrinsic interest in the different types of Helly theorems, these new types of Helly theorems are useful for getting linear time solutions for various optimization problems as is shown in [12-14]. For this, they define a new framework, DLP-type (Discrete Linear Programming type), and provide algorithms that solve in randomized linear time fixed-dimensional DLP-type problems. Furthermore, they study the relations between the LP-type and DLP-type models and the various new types of Helly theorems. They show that every lexicographic (continuous) Helly theorem yields a linear time solution to its corresponding optimization problem. Moreover, they show that many discrete Helly theorems yield linear time solutions to their corresponding optimization problems. Last, they apply these results in order to get the first randomized linear time solutions to problems such as the discrete $p$-center on the real line, the discrete weighted 1 -center problem in $\mathbb{R}^{d}$ with $l_{\infty}$ norm, the standard (continuous) problem of finding a line transversal for a totally separable set of planar convex objects, a discrete version of the problem of finding a line transversal for a set of axis-parallel planar rectangles, and the (planar) lexicographic rectilinear $p$-center problem for $p=1,2,3$.

Organization of the Paper In Sect. 2 we give discrete and lexicographic Helly theorems. We review in passing the standard (i.e., non-lexicographic continuous) Helly theorems which they generalize. We study the relations between the different types of Helly theorems in Sect. 3. In Sect. 4 we define the notions of "abstract problems" and "solution defining sets" and show how they can be used to prove Helly Theorems. In Sect. 5 we prove various discrete and lexicographic Helly theorems mentioned throughout the paper. Concluding remarks close the paper. 


\section{Discrete and Lexicographic Helly Theorems}

In this section we review previous non-lexicographic continuous Helly theorems and present the notion of discrete and lexicographic Helly theorems. We state many new such theorems. As pointed out in the introduction, lexicographic Helly theorems have algorithmic applications: [12-14] show that every lexicographic (continuous) Helly theorem yields a linear time solution to its corresponding optimization problem, and that many lexicographic discrete Helly theorems yield linear time solutions to their corresponding optimization problems.

\subsection{Generalizations of Helly's Theorem}

We start by reviewing the lexicographic version of Helly's Theorem.

Theorem 2.1 (Lexicographic Helly's Theorem) Let $H$ be a finite family of convex sets in $\mathbb{R}^{d}$. For every $x \in \mathbb{R}^{d}$, if every $d+1$ or fewer members of $H$ have a common point which is not lexicographically greater than $x$, then there is a point common to all members of $H$ which is also not lexicographically greater than $x$.

This theorem is a folklore (it derives directly from Helly's Theorem and Lemma 8.1.2 in [19]). For the sake of completeness we give here a simple proof. We need first a few definitions.

For every totally ordered set $\Lambda$ and $d \in \mathbb{N}$ we impose a lexicographic order on $\Lambda^{d}$ such that for any $x=\left(x_{1}, \ldots, x_{d}\right), y=\left(y_{1}, \ldots, y_{d}\right) \in \Lambda^{d}$ we say that $x<_{L} y(x$ is lexicographically smaller than $y$ (lsmaller, in short)) if either $x_{1}<y_{1}$ or there exists $d \geq k>1$ such that $x_{i}=y_{i}$ for $i=1,2, \ldots, k-1$, and $x_{k}<y_{k}$. We say that $x \geq_{L} y$ if $x<_{L} y$ does not hold. The following definition will be useful to us:

Definition 2.2 Let $\Lambda$ be a totally ordered set and $d \in \mathbb{N}$. For every $X \subseteq \Lambda^{d}$ and $x \in \Lambda^{d}$ let $X_{x}=\left\{x^{\prime} \in X \mid x^{\prime} \leq_{L} x\right\}$ and let $X^{x}=\left\{x^{\prime} \in X \mid x^{\prime} \geq_{L} x\right\}$.

We note that if $X$ is a convex set then for every $x \in X, X_{x}$ and $X^{x}$ are convex sets as well.

Proof of Theorem 2.1 Let $H_{x}=\left\{\mathbb{R}_{x}^{d} \cap h \mid h \in H\right\}$, so $H_{x}$ is a finite family of convex sets in $\mathbb{R}^{d}$. We conclude the proof by applying Helly's Theorem on $H_{x}$ and observing that $\bigcap H_{x} \neq \varnothing$ if and only if $H$ intersects at a point which is not lexicographically greater than $x$.

We note that the proof stated above fails when $H$ is an infinite family of compact convex sets, since the convex sets in the corresponding infinite family $H_{x}$ are not necessarily compact. This is not surprising, since the Lexicographic Helly's Theorem cannot be extended to infinite families of compact convex sets, as seen in the example below.

Example 2.3 Let $H=\left\{\left[\left(-\frac{1}{n}, 1\right) ;(0,1)\right] \mid n=1,2, \ldots\right\}$ be a set of infinitely many compact horizontal intervals of length $1, \frac{1}{2}, \ldots, \frac{1}{n}, \ldots$ Let $x=(0,0) \in \mathbb{R}^{2}$. Every 
finite subset of $H$ contains a point with negative $x$-coordinate, i.e., a point which is lsmaller than $(0,0)$. The only point common to all intervals in $H$ is $(0,1)$, which is lgreater than $(0,0)$.

By applying Observation 3.3 below, the example above demonstrates that the class of problems for which there exists a Helly theorem strictly contains the one for which there exists a lexicographic Helly theorem.

We next formally define discrete Helly-type theorems. Let $D$ be a set of elements (d-elements), $S$ be a set of elements (s-elements), and let $\mathcal{G}$ and $\mathcal{L}$ be predicates on elements of $2^{D} \times 2^{S}$. A discrete Helly theorem is a result of the form:

There is a constant $k_{D}$ such that for all subsets $S^{\prime} \subseteq S, D^{\prime} \subseteq D: \mathcal{L}\left(D^{\prime}, S^{\prime}\right)$ if and only if $\mathcal{L}\left(D^{\prime \prime}, S^{\prime}\right)$ holds for every $D^{\prime \prime} \subseteq D^{\prime}$ with $\left|D^{\prime \prime}\right| \leq k_{D}$,

and

There is a constant $k_{S}$ such that for all subsets $D^{\prime} \subseteq D, S^{\prime} \subseteq S: \mathcal{G}\left(D^{\prime}, S^{\prime}\right)$ if and only if $\mathcal{G}\left(D^{\prime}, S^{\prime \prime}\right)$ holds for every $S^{\prime \prime} \subseteq S^{\prime}$ with $\left|S^{\prime \prime}\right| \leq k_{S}$.

The least such constant $k_{D}$ is called the d-Helly number or the discrete Helly number of $(D, S)$ with respect to the predicate $\mathcal{L}$. Similarly, the least such constant $k_{S}$ is called the $s$-Helly number of $(D, S)$ with respect to the predicate $\mathcal{G}$. We say that $(D, S)$ has Helly number $\left(k_{D}, k_{S}\right)$ with respect to $\mathcal{L}$ and $\mathcal{G}$.

We say that the d-Helly number of $(D, S)$ with respect to $\mathcal{L}$ is unbounded $(\infty)$ when

For every $S^{\prime} \subseteq S$ and for every constant $k$ there is a finite $D_{k} \subseteq D$, such that for every $F \subset D_{k}$ with $|F| \leq k, \mathcal{L}\left(F, S^{\prime}\right)$ is true and $\mathcal{L}\left(D_{k}, S^{\prime}\right)$ is false.

The definition of an unbounded s-Helly number is analogous.

Note that the (somewhat strange) definition of discrete Helly theorems is a natural generalization of continuous Helly theorems. By choosing $\mathcal{G}$ to be the trivial predicate which always returns "true" and $\mathcal{L}$ to satisfy $\forall D^{\prime} \subseteq D \forall S^{\prime}, S^{\prime \prime} \subseteq S$, $\mathcal{L}\left(D^{\prime}, S^{\prime}\right)=\mathcal{L}\left(D^{\prime}, S^{\prime \prime}\right)$ we get that the class of discrete Helly theorems contains the class of (standard) Helly theorems.

We note that for general convex sets the Discrete Helly's Theorem mentioned in the introduction has an unbounded d-Helly number. To see this, let $S$ consist of $n$ points in convex position, and let $D$ be the family consisting of the convex hulls of any $n-1$ of the points.

As far as we are aware of, the theorem of Doignon [5] is the only example in the literature for a discrete Helly theorem.

Theorem 2.4 [5] Let $H$ be a finite family of at least $2^{d}$ convex sets in $\mathbb{R}^{d}$. If every $2^{d}$ or fewer members of $H$ have a common point with integer coordinates, then there is a point with integer coordinates common to all members of $H$.

This theorem is in fact a discrete Helly theorem where $D=H$ and $S$ is the set of all points in the integer lattice in the plane: for all $D^{\prime} \subseteq D$ and $S^{\prime} \subseteq S, \mathcal{L}\left(D^{\prime}, S^{\prime}\right)=$ true if and only if there exists a point in $S^{\prime}$ which lies in the intersection of the sets in $D^{\prime}$, and $\mathcal{G}\left(D^{\prime}, S^{\prime}\right)=$ true if and only if $\mathcal{L}\left(D^{\prime}, S^{\prime}\right)=$ false. Its Helly number is $\left(2^{d}, 1\right)$. In what follows, we will be mostly interested in d-Helly numbers. For simplicity, whenever it is clear from the context, we will call the d-Helly number of $(D, S)$ with respect to the predicate $\mathcal{L}$, the discrete Helly number of $(D, S)$, and denote it by $h^{*}$. 
Combining the above theorem with the Lexicographic Helly's Theorem, Theorem 2.1, we get the following theorem:

Theorem 2.5 Let $H$ be a finite family of at least $2^{d}$ convex sets in $\mathbb{R}^{d}$. For every $x \in \mathbb{R}^{d}$, if every $2^{d}$ or fewer members of $H$ have a common point $x^{\prime} \in \mathbb{Z}^{d}$ with $x^{\prime} \leq_{L} x$, then there is a point $p \in \mathbb{Z}^{d}$ common to all members of $H$ with $p \leq_{L} x$.

The Helly number remains $2^{d}$ even if we restrict $H$ to be a family of halfspaces in $\mathbb{R}^{d}$. The proof of this theorem is similar to the one of Theorem 2.1.

We conclude this subsection by noting that Helly's Theorem can be viewed as either the non-lexicographic version of Theorem 2.1, or the continuous version of Theorem 2.4, or the non-lexicographic continuous version of Theorem 2.5.

\subsection{Helly Theorems for $p$-Pierceability}

In this subsection we consider Helly-type theorems related to rectilinear $p$-piercing problems. In the (non-lexicographic) rectilinear p-piercing problem $(p \in \mathbb{N})$ we are given a finite set $B$ of compact boxes in $\mathbb{R}^{d}$ with edges parallel to the coordinate axes and decide whether there exists a set $A=\left\{a_{1}, a_{2}, \ldots, a_{p}\right\}$ of $p$ points in $\mathbb{R}^{d}$, such that for every box $b \in B$ there exists $i$ such that box $b$ is pierced by (i.e., contains) point $a_{i}$. If such a set $A$ exists we say that $B$ is $p$-pierceable and that $A$ p-pierces $B$. (We note that $B$ is 1-pierceable if and only if all the boxes in $B$ intersect.) The Helly number related to this problem is the least number $h=h(d, p)$ such that $B$ is $p$-pierceable if each $B^{\prime} \subseteq B$ with $\left|B^{\prime}\right| \leq h$ is $p$-pierceable. In 1982 Danzer and Grünbaum proved the following theorem for a (not necessarily finite) family of compact axis-parallel boxes.

\section{Theorem 2.6 [3]}

$$
\begin{aligned}
& \text { (i) } h(d, 1)=2 \text { for all } d \in \mathbb{N} ; \\
& \text { (ii) } h(1, p)=p+1 \text { for all } p \in \mathbb{N} ; \\
& \text { (iii) } h(d, 2)= \begin{cases}3 d & \text { for odd } d \\
3 d-1 & \text { for even } d ;\end{cases} \\
& \text { (iv) } h(2,3)=16 ; \\
& \text { (v) } h(d, p)=\infty \quad \text { for } d \geq 2, p \geq 3 \text { and }(d, p) \neq(2,3) .
\end{aligned}
$$

It is easy to change their proof slightly such that the theorem holds also for finite families of open axis-parallel boxes. If all the boxes are convex, but some of the boxes are neither closed nor open, the theorem does not necessarily hold. Consider for example case (i) for $d=2$ with an instance consisting of the following three boxes. Let $b_{1}$ be the closed square with edge lengths 2 and bottom-left corner at the origin, minus the interval $((1,0) ;(2,0)]$. Clearly $b_{1}$ is convex. Let $b_{2}$ be a closed rectangle with horizontal length 2 and vertical length 1 , whose upper-left corner is the origin, minus the same interval (so $b_{1}$ and $b_{2}$ are both convex and intersect in the closed interval $[(0,0) ;(1,0)])$. Let $b_{3}$ be a closed square as drawn in Fig. 1. Every two rectangles intersect but all three don't. 
Fig. 1 Every 2 rectangles intersect but all 3 don't

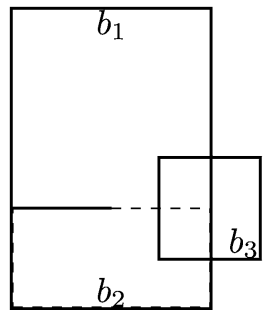

We note that case (i) in the theorem above is a special case of Helly's theorem, where the Helly number is reduced from $d+1$ to 2 since the convex sets are restricted to be closed axis-parallel boxes.

We now consider a lexicographic version of Theorem 2.6. In the lexicographic rectilinear $p$-piercing decision problem ( $p$-lpiercing decision problem, in short) we are given a finite set $B$ of closed axis-parallel boxes in $\mathbb{R}^{d}$ and a $p$-tuple $A=\left(a_{1}, \ldots, a_{p}\right)$ of $p$ points in $\mathbb{R}^{d}$ with $a_{i} \leq_{L} a_{j}, \forall i<j$. We need to decide whether there exists a $p$-tuple $A^{\prime}=\left(a_{1}^{\prime}, a_{2}^{\prime}, \ldots, a_{p}^{\prime}\right)$ such that $\left\{a_{1}^{\prime}, a_{2}^{\prime}, \ldots, a_{p}^{\prime}\right\} p$-pierces $B$ and $A^{\prime} \leq_{L} A$. If such a $p$-tuple $A^{\prime}$ exists we say that $B$ is $A$-p-pierceable and call $A^{\prime}$ a p-piercing vector of $B$.

In the lexicographic rectilinear $p$-piercing optimization problem ( $p$-lpiercing optimization problem, in short) we are given a finite set $B$ of closed boxes in $\mathbb{R}^{d}$ with edges parallel to the coordinate axes and need to find the lexicographically least $p$ tuple $A$ such that $A p$-pierces $B$. If no such $p$-tuple exists we return a special symbol $\infty$.

The Helly-type theorem related to these problems is about the least $h_{L}=h_{L}(d, p)$ such that for all $A, B$ is $A$-p-pierceable if each $B^{\prime} \subseteq B$ with $\left|B^{\prime}\right| \leq h_{L}$ is $A$-ppierceable.

\section{Theorem 2.7}

$$
\begin{aligned}
& \text { (i) } h_{L}(d, 1)=\max (2, d) \text { for all } d \in \mathbb{N} \text {; } \\
& \text { (ii) } h_{L}(1, p)=p+1 \text { for all } p \in \mathbb{N} ; \\
& \text { (iii) } h_{L}(2,2)=6 \\
& \text { (iv) } 16 \leq h_{L}(2,3) \leq 34 ; \\
& \text { (v) } h_{L}(d, p)=\infty \text { for } d \geq 2, p \geq 3 \text {, and }(d, p) \neq(2,3) .
\end{aligned}
$$

We give proofs of cases (i), (ii) and (v) in Sect. 5.1. The proofs of cases (iii) and (iv) are very technical and are given in [12]. Comparing this theorem with Theorem 2.6, we note that $h_{L}(3,1)>h(3,1)=2$ as demonstrated in the example below.

Example 2.8 Let us consider the 3 axes parallel cubes centered at $(0,0,1) ;(0,1,0)$ and $(1,0,0)$ with radius 1 (i.e., the edges are of length 2$)$. Choosing $x=(0,0,-0.5)$, we get that any pair of cubes has a common point which is lsmaller than $x$, but the lsmallest point common to all 3 is $(0,0,0)$.

We also note that $h_{L}(2,2)>h(2,2)=5$ as seen in: 
Fig. 2 An instance of the 2-piercing problem which demonstrates that $h_{L}(2,2)>h(2,2)$

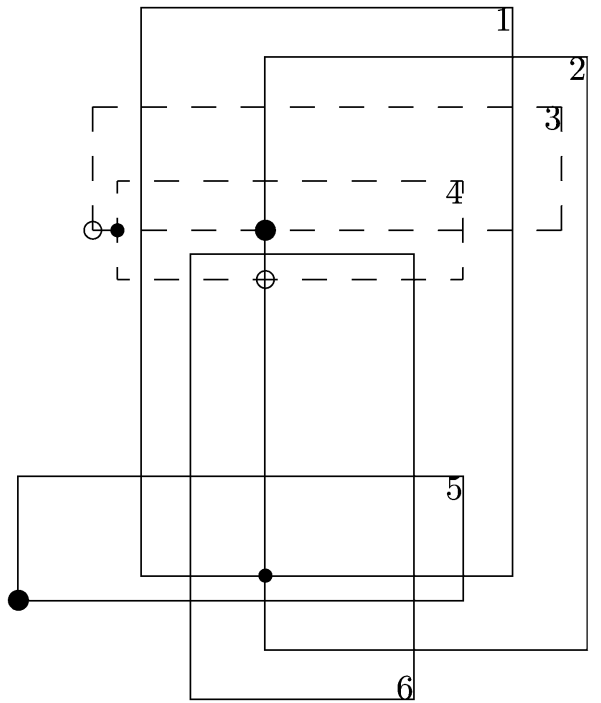

Example 2.9 Let $B$ be the set of rectangles $=\{1,2,3,4,5,6\}$ as depicted in Fig. 2 . The solution of the 2-lpiercing problem on $B$ is the two small black points in Fig. 2. The solution of the 2-lpiercing problem on each of the 6 possible subsets of 5 rectangles is lexicographically smaller than the one on $B$. We note that the upper small black point is the solution of the 1-lpiercing problem on $\{3,4\}$, and the lower small black point is the solution of the 1-lpiercing problem on $\{1,2\}$. Hence the solution of the 2-lpiercing problem on subsets of 5 rectangles not containing $\{1,2,3,4\}$ is lsmaller than the one on $B$. Also the solution on the subset of the 5 rectangles not containing 5 (the two small white points) and the solution on the subset of the 5 rectangles not containing 6 (the two big black points) are both lsmaller than the one on $B$.

We next consider discrete versions of rectilinear $p$-piercing and rectilinear $p$-lpiercing problems. The input for these problems includes a finite set $D$ of closed boxes and a finite set $S$ of points. The question is whether the rectangles in $D$ are $p$-pierceable by points in $S$. We are interested to find the least number $h^{*}=h^{*}(d, p)$ such that $D$ is $p$-pierceable by points in $S$ if each $D^{\prime} \subseteq D$ with $\left|D^{\prime}\right| \leq h^{*}$ is $p$-pierceable by points of $S$. Moreover, we are interested to find the least number $h_{L}^{*}=h_{L}^{*}(d, p)$ such that for all $p$-tuple $A$ and $S, D$ is $A$-p-pierceable by points in $S$ if each $D^{\prime} \subseteq D$ with $\left|D^{\prime}\right| \leq h_{L}^{*}$ is $A$-p-pierceable by points of $S$. We have the following discrete version of Theorem 2.6 and Theorem 2.7.

\section{Theorem 2.10}

(i) $h^{*}(d, 1)=h_{L}^{*}(d, 1)=2 d$ for all $d \in \mathbb{N}$;

(ii) $h^{*}(1, p)=h_{L}^{*}(1, p)=p+1$ for all $p \in \mathbb{N}$;

(iii) $h^{*}(d, p)=h_{L}^{*}(d, p)=\infty$ for $d \geq 2, p \geq 2$. 
Fig. 3 Every 3 rectangles intersect in a black point but all 4 don't

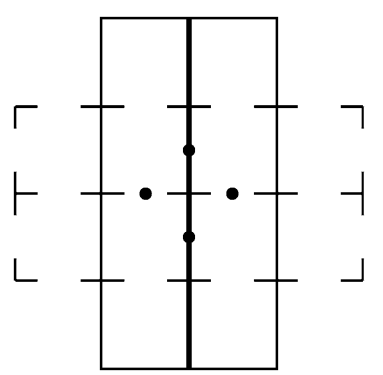

We prove this theorem in Sect. 5.1. Regarding case (i), we note that $h^{*}(d, 1)>$ $h(d, 1)=2$, as seen in:

Example 2.11 Let us consider the 4 rectangles $(|D|=4)$ and 4 points $(|S|=4)$ as drawn in Fig. 3. Each 3 rectangles intersect in exactly one of the black points but all 4 do not intersect in any of the 4 points. This proves that the d-Helly number is greater than 3 . This example can be easily extended to any dimension $d$ to get that the d-Helly number is greater than $2 d-1$.

\subsection{Helly Theorems for Line Transversals}

Some of the most interesting Helly theorems are about line transversals for a family $H$ of convex sets in the plane (a line is said to be a line transversal for $H$ if it intersects every set in $H$ ). Santaló [20] showed in 1940 that in general the Helly number is unbounded. Hadwiger, Debrunner and Klee [16] showed subsequently that a Helly number does not exist even if the convex sets are restricted to be pairwise disjoint. It soon became clear that "Helly theorems with transversals" cannot be expected unless rather severe restrictions are placed on either the shapes or the mutual position of the sets considered. A typical condition, for instance, is that the convex sets are rectangles. Another typical condition is that the sets are pairwise disjoint and translates of one another. We will concentrate here solely on line transversal problems in the plane. For the ease of presentation, we formulate the Helly theorems below for finite families of closed sets.

Theorem 2.12 Let $D$ be a family of rectangles in the plane with edges parallel to the axes, let $S$ be a set of reals (line directions), and let $a^{\prime}, b^{\prime}$ be real numbers. The discrete and lexicographic Helly numbers with respect to the property of $D$ admitting a line transversal $y=a x+b$ with $a \in S$ and $(a, b) \leq_{L}\left(a^{\prime}, b^{\prime}\right)$ are $h_{L}^{*}=h^{*}=8$ and $h_{L}=6$.

The corresponding standard Helly number is $h=6$, due to the theorem of Santaló [20]. We deduce Theorem 2.12 from:

Theorem 2.13 Let $D$ be a family of rectangles in the plane with edges parallel to the axes, let $S$ be a set of non-negative reals (line directions), and let $a^{\prime}, b^{\prime}$ be real numbers. The discrete and lexicographic Helly numbers with respect to the property 
of $D$ admitting a line transversal $y=a x+b$ with non-negative slope $a \in S$ and $(a, b) \leq_{L}\left(a^{\prime}, b^{\prime}\right)$ are $h_{L}^{*}=h^{*}=4$ and $h_{L}=3$.

The corresponding standard Helly number is $h=3$, due to a theorem of Hadwiger and Debrunner [15]. (A translation of their proof to English is given in [16].) Their proof relies on a transformation to Helly's Theorem, and in fact is valid also when $H$ is a finite family of open rectangles.

When $H$ is a family of pairwise disjoint translates of a disc, Danzer [2] proved that the Helly number is 5. Grünbaum [10] proved that if the rectangles in $H$ are axisparallel, pairwise disjoint and translates of one another, then the Helly number 6 can be replaced by 5 . Moreover, Grünbaum conjectured that the Helly number remain 5 when $H$ is relaxed to be a family of pairwise disjoint translate of a (general) convex object. In 1989, some 31 years later, Tverberg [21] proved his conjecture.

Theorem 2.14 Let $H$ be a set of pairwise disjoint translates of a compact set $O$ in the plane. The minimal $k \in \mathbb{N}$ such that if any $k$ or fewer translates from $H$ are met by a common line, then some line meets all sets of $H$ is:

$\begin{array}{llll}\text { (i) } & {[20]} & 3 & \text { when } O \text { is a segment; } \\ \text { (ii) } & {[2]} & 5 & \text { when } O \text { is a disc; } \\ \text { (iii) } & {[10]} & 5 & \text { when } O \text { is a rectangle; } \\ \text { (iv) } & {[21]} & 5 & \text { when } O \text { is convex. }\end{array}$

We give the following lexicographic-discrete version for this theorem:

Theorem 2.15 Let $D$ be a set of at least $k$ pairwise disjoint translates of a compact set $O$ in the plane, and let $S$ be a set of reals (line directions). For every pair of reals $\left(a^{\prime}, b^{\prime}\right)$, the minimal $k \in \mathbb{N}$ such that if any $k$ translates from $D$ are met by a common line $y=a x+b$ with $a \in S$ and $(a, b) \leq_{L}\left(a^{\prime}, b^{\prime}\right)$, then $D$ does as well is:

(i) 4 when $O$ is a vertical segment;

(ii) 7 when $O$ is a rectangle with edges parallel to the axes.

The Helly numbers of the non-lexicographic discrete versions of the statement above remain the same, and the Helly numbers of the lexicographic continuous versions of the statement above are 3 and 5, respectively.

Considering a variant of the discrete line transversal problem, where $S$ is a finite set of lines (and not of line directions) we have:

Theorem 2.16 Let $O$ be either a compact segment, or a rectangle in the plane. For every positive integer $k$, there exists a finite set $D$ of pairwise disjoint translates of $O$ and a set $S$ of lines, such that every $k$ translates from $D$ are met by a common line in $S$ and no line in $S$ meets all translates of $D$.

Proof We start with a construction for the case where $O$ is an interval. For every $i \in \mathbb{N}$ we construct an instance $(D, S)$ of the problem where $|D|=|S|=2 i+1, D$ 
Fig. 4 Every 6 intervals admit a line transversal but not all 7

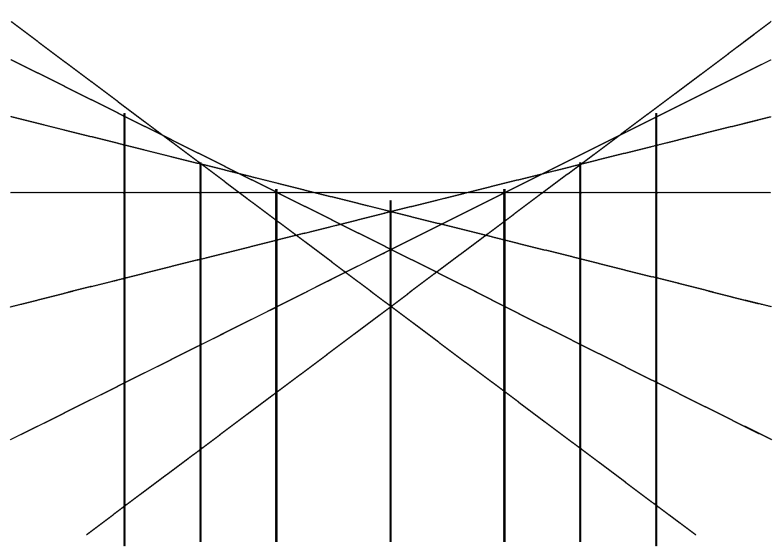

does not admit a line transversal in $S$ and any of its proper subsets does. In Fig. 4 we have $i=3, D$ consists of $3 * 2+1=7$ parallel vertical intervals, and $S$ contains the 7 thin lines. Looking at the upper envelope of the lines it is easy to see that every 6 intervals admit a line transversal but not all 7 .

So the Helly number corresponding to the problem is infinite, when $O$ is an interval. Changing the segments in the above example into very narrow rectangles proves the infinity of the Helly number for the case of rectangles as well.

In 1957 Hadwiger [11] realized that if a family of disjoint convex sets have a line transversal, then this transversal meets the sets in a specific order (if we take the line to be directed). Thus, if the family $H=\left\{h_{1}, \ldots, h_{n}\right\}$ is met by a directed line $\ell$, then $\ell$ meets the family in some order $h_{\pi(1)}, \ldots, h_{\pi(n)}$ which is described by the permutation $\pi$. We then say that $\ell$ meets $H$ consistently with the order corresponding to $\pi$. We say that a non-directed line $\ell^{\prime}$ meets $H$ consistently with the order corresponding to $\pi$ if at least one of the two possible directed lines of $\ell^{\prime}$ meets $H$ consistently with the order. Let now $H$ be a (not necessarily pairwise disjoint) ordered family of convex sets. We say that a directed line $\ell$ meets $H$ consistently with the order if it meets every ordered pair of disjoint sets from $H$ in that order.

Theorem 2.17 Let $D$ be a linearly ordered family of convex sets, let $S$ be a set of reals, and let $a^{\prime}$ and $b^{\prime}$ be real numbers. If every subfamily from $D$ of at most 4 sets is met by a line $y=a x+b$ with $a \in S$ and $(a, b) \leq_{L}\left(a^{\prime}, b^{\prime}\right)$, consistently with the order, then $D$ admits a line transversal $y=a^{\prime \prime} x+b^{\prime \prime}$ with $a^{\prime \prime} \in S$ and $\left(a^{\prime \prime}, b^{\prime \prime}\right) \leq_{L}$ $\left(a^{\prime}, b^{\prime}\right)$. The corresponding non-lexicographic discrete Helly number remains 4 and the lexicographic continuous Helly number is 3.

The corresponding standard Helly number is $h=3$, due to a theorem by Wenger [23]. We note in passing that Wenger's theorem is a stronger version of the much older Hadwiger's Transversal Theorem [11] that has the same Helly number 3, but requires the objects in $D$ to be pairwise disjoint.

We also note that this Helly-type theorem is slightly different from the ones studied so far in the sense that the property $D$ is required to satisfy (admitting a line transversal $y=a^{\prime \prime} x+b^{\prime \prime}$ with $a^{\prime \prime} \in S$ and $\left.\left(a^{\prime \prime}, b^{\prime \prime}\right) \leq_{L}\left(a^{\prime}, b^{\prime}\right)\right)$ is weaker than the 
property each subset of $D$ of at most 4 sets is required to satisfy (the line transversal should be in addition consistent with the order). If we require the line transversal for all the sets in $D$ to meet them consistently with the order we get the following theorem.

Theorem 2.18 Let $D$ be a linearly ordered family of pairwise disjoint convex sets, let $S$ be a set of reals, and let $a^{\prime}, b^{\prime}$ be real numbers. The discrete and lexicographic Helly numbers with respect to the property of $D$ admitting a line transversal $y=$ $a x+b$ with slope $a \in S$ and $(a, b) \leq_{L}\left(a^{\prime}, b^{\prime}\right)$ that is consistent with the order are $h_{L}^{*}=h^{*}=h_{L}=4$.

The corresponding standard Helly number is $h=4$, and was proved independently by Tverberg [22] and Wenger [23]. Hadwiger's Transversal Theorem can be viewed as a "weaker" version of the theorems of Tverberg and Wenger since it does not require the line transversal for all sets in $D$ to meet them consistently with the order.

It is not always clear what should be the linear ordering of $H$. A case where this ordering is obvious is when $H$ consists of parallel line segments. Thus, Theorem 2.15(i) is a special case of Theorem 2.17. Another such obvious case is:

Definition 2.19 Let $H$ be a family of sets in the plane. We say that $H$ is totally separable if there exists a direction such that each line in this direction intersects at most one set from $H$.

Note that whenever $H$ is totally separable then there exists a linearly ordered set of pairwise disjoint parallel strips in the plane such that each strip contains exactly one set from $H$, and that any line transversal to any subset $H^{\prime} \subset H$ must meet the sets in $H^{\prime}$ in that order.

A corollary of Theorem 2.17 is:

Corollary 2.20 Let $D$ be a totally separable family of convex sets, let $S$ be a set of line directions, and let $a^{\prime}, b^{\prime}$ be real numbers. The discrete and lexicographic Helly numbers with respect to the property of $D$ admitting a line transversal $y=a x+b$ with slope $a \in S$ and $(a, b) \leq_{L}\left(a^{\prime}, b^{\prime}\right)$ are $h_{L}^{*}=h^{*}=4$ and $h_{L}=3$.

The corresponding standard Helly number is $h=3$ and was originally proved independently by Klee [18] and Grünbaum [9]. Grünbaum showed that this case holds for finite families of open convex sets as well [9].

\section{The Relations between the Different Types of Helly Theorems}

In this section we study the relations between the 4 different kinds of Helly numbers.

First, we show in Sect. 3.1 that $h \leq h_{L}$. In Sect. 3.2 we next show that $h^{*} \leq h_{L}^{*}$. In the last subsection we show that for every d-element set $D$, there exists an s-element set $S$, such that the corresponding Helly numbers with respect to intersection satisfy $h \leq h_{L} \leq h^{*} \leq h_{L}^{*}$. We also study the sensitivity of the Helly numbers under taking subsets, and show that they are monotone nonincreasing under taking subsets of $D$, and not necessarily monotone under taking subsets of $S$. 


\subsection{Continuous Case}

The term Helly-type theorem is often used to describe a larger class of theorems, including ones in which the fact that every subfamily has some property $\mathcal{P}$ implies that the whole family has some other property $\mathcal{Q}$. With the exception of Theorem 2.17, we will not be concerned with this larger class. We are interested in a particular subclass of Helly-type theorems, in which the objects in $H$ are subsets of a set $X$ which we call a ground set, and $\mathcal{P}(G)$ is the intersection predicate, i.e., $\forall G \subseteq H, \mathcal{P}(G)$ is that the sets in $G$ intersect in a common point. We call the subsets of $H$ constraints. We write $\bigcap G$ for $\{x \in X \mid x \in h$, $\forall h \in G\}$, and we say that the family of sets intersects when $\bigcap G \neq \emptyset$, that is, when $\mathcal{P}(G)$ is true.

We need some more notation. A set system is a pair $(X, H)$, where $X$ is a set and $H$ is a family of subsets of $X$. We say $(X, H)$ is a Helly system if there exists a finite integer $k$ such that $H$ has Helly number $k$ with respect to the intersection predicate $\mathcal{P}$. The natural computational problem associated with a Helly system is, given a subset $G \subseteq H$, return a point $x \in \bigcap G$, or show that $\bigcap G=\emptyset$.

Most Helly theorems can be restated in terms of the intersection predicate. For instance, let us consider the following theorem:

Theorem 3.1 (Radius Theorem) A family $H$ of points in the Euclidean d-dimensional space $E^{d}$, is contained in a unit ball if and only if every $d+1$ or fewer points from $H$ are contained in a unit ball.

Here the family of objects is the set of points in $E^{d}$, the predicate is that a subfamily is contained in a (closed) unit ball, and $k=d+1$. In fact, the Radius Theorem is a corollary of Helly's theorem proper. To see that we apply the following duality transformation. We transform every point $h \in H$ into the set $\mathcal{D}(h)$ of centers of unit balls containing $h$. In this way $\mathcal{D}(h)$ is a unit ball centered at $h$. Let $\mathcal{D}(H)=\{\mathcal{D}(h) \mid h \in H\}$. From the definition of this duality transformation we get that the points in $H$ are contained in a unit ball if and only if the unit balls in $\mathcal{D}(H)$ have non-empty intersection (see Fig. 5). Since the objects in $\mathcal{D}(H)$ are convex sets, and by using Helly's Theorem, the Radius Theorem follows. We note in passing that it is easy to show that the discrete version of the Radius Theorem has unbounded Helly number.

Definition 3.2 Let $\Lambda$ be a totally ordered set. A Helly system with lexicographic Helly number $l$ is a set system $(X, H)$ where $X \subseteq \Lambda^{d}$ for some positive integer $d$, such that for any $x \in X,\left(X,\left\{h \cap X_{x} \mid h \in H\right\}\right)$ is a Helly system with Helly number $l$.

Fig. 5 The 3 points on the left side are contained in a unit ball if and only if the 3 unit balls on the right side intersect
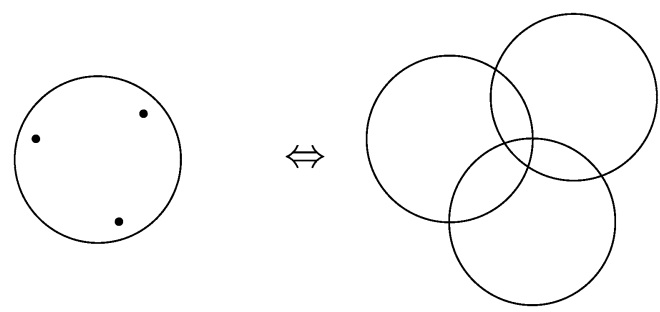
This means that for any $x \in X$, whenever every $l$ or less elements of $H$ have a common point which is not lgreater than $x$, we get that all elements of $H$ have a common point which is not lgreater than $x$.

Comparing between a Helly system with a lexicographic Helly number and its corresponding non-lexicographic Helly system we get the following observation:

Observation 3.3 The lexicographic and standard Helly numbers of any Helly system satisfy $h \leq h_{L}$.

Proof Suppose first that $(X, H)$ is a (non-lexicographic) Helly system with a finite Helly number $h$. From the minimality of $h$ there is a subset $G \subseteq H,|G|=h$ such that $\bigcap G=\emptyset$ and for every proper subset $F \subset G$ we have $\bigcap F \neq \emptyset$. For every such $F$ let $x_{F}$ be an arbitrary point in $\bigcap F$. Let

$$
x=\text { lexicographic } \max _{F \subset G}\left\{x_{F}\right\} .
$$

From the definition of $x$, every proper subset of $G$ intersects in a point which is not lgreater than $x$. Since $G$ does not intersect, we get that the lexicographic Helly number is greater than $h-1$.

Assume now that $(X, H)$ is a Helly system with unbounded Helly number. In this case there is an infinite sequence of sets $G_{k} \subset H, k \in \mathbb{N}$, such that for every $k$ and for all $F_{k} \subset G_{k}$ with $\left|F_{k}\right| \leq k$, we get that $\bigcap F_{k} \neq \emptyset$ and $\bigcap G_{k}=\emptyset$. For every such $F_{k}$ let $x_{F_{k}}$ be an arbitrary point in $\bigcap F_{k}$. Let

$$
x_{k}=\text { lexicographic } \max _{F_{k} \subset G_{k}}\left\{x_{F_{k}}\right\} \text {. }
$$

We look at an arbitrary subset $H^{\prime}$ of $H$ containing $\cup_{i} G_{i}$. From the construction, for every $k$ there is a subset $G_{k} \subset H^{\prime}$ such that $\bigcap G_{k}=\emptyset$ but any subset $F_{k} \subset G_{k}$ with $\left|F_{k}\right| \leq k$ intersects in a point not lgreater than $x_{k}$. Hence the lexicographic Helly number of $(X, H)$ is unbounded.

\subsection{Discrete Case}

Similarly to the case of (continuous) Helly theorems, we are mainly concerned with discrete Helly theorems in which $D, S$ are families of sets and $\mathcal{P}$ is the property of having non-empty intersection. A discrete set system is a triple $(X, D, S)$, where $X$ is a set and $D, S$ are families of subsets of $X$. For $D^{\prime} \subseteq D$, we write $\bigcap D^{\prime}$ for $\left\{x \in X \mid x \in h, \forall h \in D^{\prime}\right\}$. For $S^{\prime} \subseteq S$, we write $\bigcup S^{\prime}$ for $\left\{x \in X \mid \exists h \in S^{\prime}, x \in h\right\}$. Let $H=\left(D^{\prime}, S^{\prime}\right) \in 2^{D} \times 2^{S}$. We write $\bigcap H$ for $\bigcap D^{\prime} \cap\left(\bigcup S^{\prime}\right)$, i.e., the intersection of $\bigcap D^{\prime}$ with the "grid" $\cup S^{\prime}$. We say that $H$ satisfies the intersection predicate, or simply intersects, when $\bigcap H \neq \emptyset$.

Observation 3.4 The discrete set system $(X, D, S)$ is equivalent to the set system $(X, D)$ on the grid $\bigcup S$ (i.e., to the set system $(X \cap(\bigcup S), D \cap(\bigcup S))$ ), in the sense that $(D, S)$ intersects (in the discrete set system sense, i.e., $\cap D \cap(\bigcup S) \neq \emptyset)$ if and only if $D \cap(\bigcup S)$ intersects (in the set system sense, i.e., $\bigcap(D \cap(\bigcup S)) \neq \emptyset)$. 
Definition 3.5 A discrete set system $(X, D, S)$ is a discrete Helly system with Helly number $k$ if $(D, S)$ has a Helly number $(k, 1)$ with respect to $\mathcal{L}$ being the intersection predicate and $\mathcal{G}$ being the non intersection predicate, i.e., $\forall D^{\prime} \subseteq D, S^{\prime} \subseteq S$, $\mathcal{L}\left(D^{\prime}, S^{\prime}\right)=\neg \mathcal{G}\left(D^{\prime}, S^{\prime}\right)$.

Let $(X, D, S)$ be a discrete set (Helly) system. We say that $(X, D)$ is its corresponding continuous set (Helly) system, respectively. We note that it is sometimes useful to think of the set system $(X, D)$ as the discrete set system $(X, D,\{X\})$.

Regarding lexicographic discrete Helly-type theorems, we are interested in those theorems where $D$ and $S$ are families of subsets from $X, x \in X$, and the property in question is of having non-empty intersection with $S \cap X_{x}$.

Definition 3.6 A discrete Helly system with lexicographic Helly number ld is a discrete set system $(X, D, S)$ such that for any $x \in X,\left(X,\left\{d \cap X_{x} \mid d \in D\right\},\left\{s \cap X_{x} \mid\right.\right.$ $s \in S\})$ is a discrete Helly system with Helly number $l d$.

This means that for any $x \in X$, whenever every $l d$ or less elements of $D$ have a common point in $S$ which is not lgreater than $x$, we get that all elements of $D$ have a common point in $S$ which is not lgreater than $x$.

Comparing lexicographic discrete Helly systems with their corresponding nonlexicographic discrete Helly systems we get the following monotone structure

Observation 3.7 The lex-discrete and discrete Helly numbers of any discrete Helly system satisfy $h^{*} \leq h_{L}^{*}$.

The proof of this observation is similar to the one of Observation 3.3.

Comparing the 4 different types of Helly numbers, we have the following result.

Observation 3.8 For every set $X$ of elements and a family $D$ of subsets of $X$, there exists a family $S$ of subsets of $X$, such that the Helly numbers corresponding to the Helly system $(X, D)$ and discrete Helly system $(X, D, S)$ satisfy $h \leq h_{L} \leq h^{*} \leq h_{L}^{*}$.

Proof We first show that $h_{L} \leq h^{*}$. Considering the Helly system $(X, D)$, suppose first that $h_{L}$ is finite. By the minimality of $h_{L}$ there exists a family $D^{\prime} \subseteq D$ and a point $x \in X$, such that each subset $D^{\prime \prime} \subseteq D^{\prime}$ with $\left|D^{\prime \prime}\right| \leq h_{L}-1$ has a non-empty intersection point $p\left(D^{\prime \prime}\right) \leq_{L} x$, but $D^{\prime}$ does not have such an intersection point. In this way $h_{L}$ serves as a lower bound for the discrete Helly number in the discrete Helly system $(X, D, S)$, where $S:=\left\{p\left(D^{\prime \prime}\right) \mid D^{\prime \prime} \subseteq D^{\prime}\right.$ with $\left.\left|D^{\prime \prime}\right| \leq h_{L}-1\right\}$. If $(X, D)$ is a Helly system with unbounded Helly number, there exists a point $x \in X$ and an infinite sequence of sets $D_{k} \subset D, k \in \mathbb{N}$, such that for every $k$ and for all $F_{k} \subset G_{k}$ with $\left|F_{k}\right| \leq k$, we get that $X_{x} \cap\left(\bigcap F_{k}\right) \neq \emptyset$ and $X_{x} \cap\left(\bigcap G_{k}\right)=\emptyset$. For every such $F_{k}$ let $x_{F_{k}}$ be an arbitrary point in $X_{x} \cap\left(\bigcap F_{k}\right)$, and let $S:=\bigcup_{k=1}^{\infty}\left\{x_{F_{k}}\right\}$. Then $(X, D, S)$ is a discrete Helly system with unbounded Helly number.

We conclude by using Observations 3.3 and 3.7.

The statement in Observation 3.8 does not necessarily hold for every family $S$ of subsets: Take for example $X=\mathbb{R}, D$ - the set of compact intervals on the real line, 
and $S=\{0\}$. Due to Helly's Theorem the standard Helly number is 2, and it is easy to see that the discrete Helly number of $(X, D, S)$ is 1 .

Comparing Theorem 2.15 with Theorem 2.16, which are both discrete versions of Theorem 2.14, we note that in Theorem 2.15 there is a bounded Helly number while in Theorem 2.16 there isn't. A possible intuitive explanation for this is as follows. In the original (continuous) Theorem 2.14, the line transversal is chosen from the infinite set of all lines. In Theorem 2.15, even though the set of allowed lines is a proper subset of the original one, it is still infinite. In Theorem 2.16 the set of allowed lines is finite. In this sense Theorem 2.15 resembles the continuous Helly theorem, Theorem 2.14, more than Theorem 2.16. In a way, we may think of Theorem 2.15 as a "weak" discrete version of Theorem 2.14, and of Theorem 2.16 as a "strong" discrete version.

In view of this, a natural question is whether Helly numbers are non-increasing under taking subsets. The next observation tells us that this is indeed the case for taking subsets of the d-element set, but not for taking subsets of the s-element set.

Observation 3.9 For every discrete Helly system $(X, D, S)$ and subset $D^{\prime} \subset D$, each of the 4 different kinds of the Helly numbers of $(X, D, S)$ is not smaller then its corresponding Helly number of $\left(X, D^{\prime}, S\right)$. Moreover, there exist a discrete Helly system $(X, D, S)$ and subsets $S_{2} \subset S_{1} \subset S$, such that $h_{2}^{*}<h_{1}^{*}>h^{*}$, where $h_{2}^{*}, h_{1}^{*}, h^{*}$ are the discrete Helly numbers of $\left(X, D, S_{2}\right),\left(X, D, S_{1}\right),(X, D, S)$, respectively.

Proof The proof of the first statement follows directly from the definitions of Helly numbers, since subsets of $D^{\prime}$ are subsets of $D$ as well.

We now give an example for the second statement. Let $D$ be the set of axis-parallel rectangles in the plane. Let

$$
\begin{aligned}
& S:=\mathbb{R}^{2}, \quad S_{1}:=\{(2 i, 2 j),(2 i+1,2 j+1) \mid i, j \in \mathbb{Z}\}, \\
& S_{2}:=\{(2 i, 2 j) \mid i, j \in \mathbb{Z}\} .
\end{aligned}
$$

Clearly $S_{2} \subset S_{1} \subset S$. Since $(X, D, S) \equiv(X, D)$ is a continuous Helly system, Theorem 2.6(i) implies that $h^{*}=2$. Due to Theorem 2.10(i) and the fact that we can realize Example 2.11 with the lattice $S_{1}$, we get that $h_{1}^{*}=4$. Last, we note that the problem of intersecting axis-parallel rectangles on the lattice $S_{2}$ decomposes into two one-dimensional problems. Hence, Theorem 2.10(ii) implies that $h_{2}^{*}=2$.

\section{From Abstract Problems to Helly Theorems}

In this section we define abstract problems and defining sets and show that there is a (discrete) Helly theorem corresponding to the constraint set of every (discrete) abstract problem, respectively. In later sections we will use this relation to prove some of the Helly theorems. Section 4.1 is based mainly on the results of Amenta [1]. In Sect. 4.2 we extend them to the discrete case. 


\subsection{Continuous Case}

We start with defining a general class of problems:

Definition 4.1 An abstract problem is a pair $(H, \omega)$ where $H$ is a finite set of elements (which we call constraints) and $\omega$ is an objective function from $2^{H}$ to some totally ordered set $\Lambda$ which contains a special maximal (minimal) element $\infty(-\infty)$, respectively. The goal is to compute $\omega(H)$.

Definition 4.2 Let $(H, \omega)$ be an abstract problem. For any subset $G \subseteq H$ we say that $F \subseteq G$ defines the solution on $G$ ( $F$ is a solution defining set of $G$ ) if $\omega(F)=\omega(G)$.

Clearly $G$ is a defining set for itself.

Definition 4.3 Let $(H, \omega)$ be an abstract problem and let $\lambda \in \Lambda$ be arbitrary. The corresponding Helly theorem is a Helly theorem where the predicate $\mathcal{P}$ is

$$
\forall G \subseteq H, \quad \mathcal{P}(G)=\text { true } \quad \Longleftrightarrow \omega(G) \leq \lambda .
$$

Generally, $H$ may have an unbounded Helly number with respect to $\mathcal{P}$. We next define a special class of abstract problems and a sufficient condition such that the corresponding Helly theorems for these abstract problems have finite Helly numbers.

Definition 4.4 Given an abstract problem $(H, \omega)$, we say that $(H, \omega)$ satisfies the Monotonicity Condition if (when we write $<, \leq,=$ etc. we mean under the ordered set $\Lambda$ ) for all $F \subseteq G \subseteq H: \omega(F) \leq \omega(G)$.

Theorem 4.5 Let $(H, \omega)$ be an abstract problem which meets the Monotonicity Condition. If for every $G \subseteq H$ there exists a solution defining set of cardinality at most $k$ then for every $\lambda \in \Lambda$ and for every $G \subseteq H, G$ has the property $\omega(G) \leq \lambda$ if and only if every $F \subseteq G$ with $|F| \leq k$ has the property $\omega(F) \leq \lambda$.

Proof Let $\omega(G) \leq \lambda$. By the Monotonicity Condition, every $F \subseteq G$ must have $\omega(F) \leq \omega(G) \leq \lambda$. Going in the other direction, there exists a solution defining set $F$ for $G$ of cardinality at most $k$, that is, $\omega(G)=\omega(F)$, with $|F| \leq k$. So if every subfamily $F$ with $|F| \leq k$ has $\omega(F) \leq \lambda$, then $\omega(G)=\omega(F) \leq \lambda$.

The theorem above says that $k$ serves as an upper bound for the Helly number of the Helly theorem corresponding to the abstract problem $(H, \omega)$. We note that Grünbaum basically uses the same idea to prove Theorem 2.14(iii) [10].

\subsection{Discrete Case}

We start with "discretizing” abstract problems:

Definition 4.6 A discrete abstract problem is a triple $(D, S, \omega)$ where $D$ and $S$ are finite sets of elements and $\omega$ is an objective function from $2^{D} \times 2^{S} \backslash\{(\emptyset, \varnothing)\}$ to some totally ordered set $\Lambda$ which contains a special maximal (minimal) element $\infty(-\infty)$, respectively. The goal is to compute $\omega(D, S)$. 
Definition 4.7 Let $(D, S, \omega)$ be a discrete abstract problem. For any $G=\left(D^{\prime}, S^{\prime}\right) \subseteq$ $2^{D} \times 2^{S}$ we say that $F=\left(D^{\prime \prime}, S^{\prime}\right) \subseteq 2^{D^{\prime}} \times 2^{S^{\prime}}$ defines the solution on $G$ ( $F$ is a solution defining set of $G)$ if $\omega(F)=\omega(G)$.

Similarly to the continuous case, $G$ is a defining set for itself.

Definition 4.8 Given a discrete abstract problem $(D, S, \omega)$, we say that $(D, S, \omega)$ satisfies the Monotonicity of Demand Condition if (when we write $<, \leq,=$ etc. we mean under the ordered set $\Lambda$ ) for all $S^{\prime} \subseteq S$ and $D^{\prime \prime} \subseteq D^{\prime} \subset D: \omega\left(D^{\prime \prime}, S^{\prime}\right) \leq \omega\left(D^{\prime}, S^{\prime}\right)$.

Definition 4.9 Let $(D, S, \omega)$ be a discrete abstract problem and $\lambda \in \Lambda$ be arbitrary. The corresponding discrete Helly theorem is the discrete Helly theorem where the predicates $\mathcal{L}$ and $\mathcal{G}$ are $\forall D^{\prime} \subseteq D, S^{\prime} \subseteq S$

$$
\mathcal{L}\left(D^{\prime}, S^{\prime}\right)=\text { true } \Longleftrightarrow \omega\left(D^{\prime}, S^{\prime}\right) \leq \lambda,
$$

and

$$
\mathcal{G}\left(D^{\prime}, S^{\prime}\right)=\text { true } \Longleftrightarrow \omega\left(D^{\prime}, S^{\prime}\right) \geq \lambda
$$

Generally $(D, S)$ may have an unbounded Helly number with respect to $\mathcal{L}$ and $\mathcal{G}$. We now "discretize" Theorem 4.5.

Theorem 4.10 Let $(D, S, \omega)$ be a discrete abstract problem which meets the Monotonicity Condition. If for every $G=\left(D^{\prime}, S^{\prime}\right) \subseteq 2^{D} \times 2^{S}$ there exists $D^{\prime \prime} \subseteq D^{\prime}$ with $\left|D^{\prime \prime}\right| \leq k$ such that $F=\left(D^{\prime \prime}, S^{\prime}\right)$ is a solution defining set of $G$, then for every $\lambda \in \Lambda$ and for every $G=\left(D^{\prime}, S^{\prime}\right) \subseteq 2^{D} \times 2^{S}, G$ has the property $\omega(G) \leq \lambda$ if and only if every $D^{\prime \prime} \subseteq D^{\prime}$ with $\left|D^{\prime \prime}\right| \leq k$ implies that $\omega\left(D^{\prime \prime}, S^{\prime}\right) \leq \lambda$.

The proof is similar to the one of Theorem 4.5 .

\section{Proving Helly Theorems}

In this section we prove some of the Helly theorems mentioned above.

\subsection{Theorems Related to $p$-pierceability}

\subsubsection{Theorem 2.7 cases (i), (ii) and (v)}

Let $B$ be a finite set of axis-parallel rectangles in $\mathbb{R}^{d}$. Let $\left(B, \omega_{p}\right)$ be an abstract problem over the totally ordered set $\mathbb{R}^{2 p} \cup\{\infty\}$, where for every $B^{\prime} \subseteq B$ and natural number $p, \omega_{p}\left(B^{\prime}\right)$ is the lexicographically smallest $p$-piercing vector which is feasible for $B^{\prime}$ (if $B^{\prime}$ is not $p$-pierceable, we set $\omega_{p}\left(B^{\prime}\right)=\infty$ ). Since adding a rectangle $b$ to $B^{\prime}$ cannot lexicographically decrease the lexicographically smallest feasible $p$-piercing vector, i.e., $\omega_{p}(B \cup\{b\}) \geq_{L} \omega_{p}(B)$, we get that $\left(B, \omega_{p}\right)$ is an abstract problem which meets the Monotonicity Condition. From Theorem 4.5 it is sufficient to find for every $B^{\prime} \subseteq B$ a solution defining set with cardinality which is bounded 
by the Helly number we want to prove. Let $A_{\text {opt }}$ be the solution of the lexicographic $p$-piercing optimization problem on $B$.

Case (i): We first show that $h_{L}(d, 1) \geq \max (2, d)$. By Observation 3.3 and Theorem 2.6(i), $h_{L}(d, 1) \geq h(d, 1)=2$. If $B$ is 1 -pierceable, for every $i=1, \ldots, d$ we project $B$ onto the $i$ 'th coordinate and thus get a one dimensional 1-piercing problem. Let $b_{i} \in B$ be a box whose projection on the $i$ 'th coordinate is an interval $\left[l_{i}, r_{i}\right]$ with the greatest $l_{i}$. Since $B$ intersects, $l_{i}$ is inside the projection of $B$ on the $i$ 'th coordinate. Let $B^{\prime \prime}=\left\{b_{1}, \ldots, b_{d}\right\}$. We observe that $A_{\mathrm{opt}}=\left(l_{1}, \ldots, l_{d}\right)$. It is possible to choose a set $B$ such that $B$ and $B^{\prime \prime}$ are $A_{\text {opt }}-1$-pierceable and there exists a sufficiently small $\epsilon>0$ such that neither $B$ nor $B^{\prime \prime}$ are $\left(l_{1}, \ldots, l_{d}-\epsilon\right)$-1-pierceable but every proper subset of $B^{\prime \prime}$ is $\left(l_{1}, \ldots, l_{d}-\epsilon\right)$-1-pierceable. Hence $h_{L}(d, 1) \geq \max (2, d)$.

We now show that $h_{L}(d, 1) \leq \max (2, d)$. If there exist 2 boxes in $B$ which are disjoint then $B$ is not 1-pierceable. If every 2 boxes of $B$ intersect then by Theorem 2.6(i) $B$ intersects, and the lexicographically smallest intersection vector is determined by $B^{\prime \prime}$ defined above. $B^{\prime \prime}$ is a solution defining set for $B$ of cardinality at most $d$. Hence $h_{L}(d, 1) \leq \max (2, d)$.

Case (ii): From Theorem 2.6(ii) and Observation 3.3 it remains to prove that $h_{L}(1, p) \leq p+1$. If there exist $p+1$ intervals in $B$ which are pairwise disjoint then $B$ is not $p$-pierceable and these $p+1$ intervals serve as a solution defining set for $B$. If every $p+1$ intervals of $B$ are $p$-pierceable then by Theorem 2.6(ii) $B$ is $p$-pierceable. We can suppose it is not $(p-1)$-pierceable. Let $b^{p} \in B$ be an interval $\left[l^{p}, r^{p}\right]$ with the greatest $l^{p}$. We look at the set of intervals which are not pierced by $l^{p}$ and define $b^{p-1}, \ldots, b^{1}$ and $l^{p-1}, \ldots, l^{1}$ recursively. Let $B^{\prime \prime}=\left\{b^{1}, \ldots, b^{p}\right\}$. $B^{\prime \prime}$ is a solution defining set for $B$ of cardinality at most $p$. Thus, we conclude that $h_{L}(1, p) \leq p+1$.

Case (v): It follows directly from Theorem 2.6(v) and Observation 3.3.

\subsubsection{Theorem 2.10}

Case (i): We first prove $h_{L}^{*}(d, 1)=2 d$. The Helly number is bounded from below by $2 d$ due to Example 2.11.

We will use Theorem 4.10. Let $\omega: 2^{D} \times 2^{S} \rightarrow \mathbb{R}^{2} \cup\{-\infty, \infty\}$ be a function such that for every $\left(D^{\prime}, S^{\prime}\right) \in 2^{D} \times 2^{S}, \omega\left(D^{\prime}, S^{\prime}\right)$ is the lexicographically smallest point in $S^{\prime}$, which is in $\bigcap D^{\prime}$, if such point exists and is bounded, $-\infty$ if $\bigcap D^{\prime} \cap S^{\prime} \neq \emptyset$ and is not bounded, and $\infty$ otherwise. The definition of $\omega$ implies that for every $x \in \mathbb{R}^{d}$ and $\left(D^{\prime}, S^{\prime}\right) \in 2^{D} \times 2^{S}$, the rectangles in $D^{\prime}$ have a common point $x^{\prime} \in S^{\prime}$ with $x^{\prime} \leq_{L} x$ if and only if $\omega\left(D^{\prime}, S^{\prime}\right) \leq_{L} x$. Moreover, since set intersection is monotone (i.e., $A \cap B \subseteq A, B),(D, S, \omega)$ is a discrete abstract problem (see Definition 4.7), which meets the Monotonicity of Demand Condition. Hence, due to Theorem 4.10, it is sufficient to show that for every $\left(D^{\prime}, S^{\prime}\right) \in 2^{D} \times 2^{S}$, there exists a solution defining set $\left(D^{\prime \prime}, S^{\prime}\right)$ with $\left|D^{\prime \prime}\right| \leq 2 d$.

If the intersection of the boxes in $D^{\prime}$ is empty, by Theorem 2.6(i) there are two boxes which do not intersect. We let $D^{\prime \prime}$ to consist of these two boxes.

Otherwise, for each coordinate $i=1, \ldots, d$, we act as follows. Let $L_{i}\left(D^{\prime}\right) \in D^{\prime}$ be a box whose projection on the $i$ 'th coordinate is an interval $\left[l_{i}, r_{i}\right]$ with the smallest $r_{i}$. 
We let $G_{i}(D) \in D$ be a box whose projection on the $i$ 'th coordinate is an interval $\left[l_{i}^{\prime}, r_{i}^{\prime}\right]$ with the greatest $l_{i}^{\prime}$. We let $D^{\prime \prime}=\left\{L_{1}\left(D^{\prime}\right), G_{1}\left(D^{\prime}\right), \ldots, L_{d}\left(D^{\prime}\right), G_{d}\left(D^{\prime}\right)\right\}$. We conclude the proof of this case by noting that since $\bigcap D^{\prime \prime}=\bigcap D^{\prime},\left(D^{\prime \prime}, S^{\prime}\right)$ is a solution defining set for $\left(D^{\prime}, S^{\prime}\right)$, and the cardinality of $D^{\prime \prime}$ is at most $2 d$, as needed.

As for proving $h^{*}(d, 1)=2 d$, note that Example 2.11 implies a lower bound of $2 d$, while Observation 3.7 gives an upper bound of $2 d$.

Case (ii): We transform every interval $h \in D$ into the maximum length interval $t(h)$ which is contained in $h$ and has endpoints in $S$. Note that $D$ is $p$-pierceable by points in $S$ if and only if $t(D)=\{t(h) \mid h \in D\}$ is $p$-pierceable. Hence, Theorem 2.6(ii) implies that $h^{*}(1, p)=p+1$. Note also that the lexicographically smallest $p$-piercing vector for $t(D)$ consists of left endpoints of intervals, and therefore of points in $S$. Hence, Theorem 2.7(ii) implies that $h_{L}^{*}(1, p)=p+1$.

Case (iii): It suffices to show that for every $k \in \mathbb{N}$, there exists a set $D$ of $2 k+1$ rectangles and a set $S$ of $2 k+1$ points such that every proper subset of $D$ is 2-pierceable by points in $S$, but $D$ is not 2-pierceable by points in $S$. To see this, let $B$ be the unit axis-parallel rectangle rotated by $\frac{\pi}{4}$ radians with center at the origin. Let $S$ be a set of $2 k+1$ points evenly placed on the border of $B$. Let $D$ be a set of $2 k+1$ axisparallel rectangles such that each rectangle contains exactly $k$ consecutive points on the border of $B$ from $S$. It is possible to construct such sets since $B$ is an axis-parallel rectangle which is rotated by $\frac{\pi}{4}$ radians. (We can choose $D$ such that each rectangle in $D$ contains exactly one of the edges of $B$.) Note that each point in $S$ pierces exactly $k$ rectangles from $D$, so $D$ is not 2-pierceable. We conclude by observing that since each rectangle in $D$ contains exactly $k$ different points from $S$, any subset of $D$ of cardinality $2 k$ is 2-pierceable by points in $S$.

\subsection{Proving Theorems Related to Line Transversals}

\subsubsection{Theorem 2.17}

We start by proving the lexicographic discrete case. We can assume that $\forall a \in S$, $a \leq a^{\prime}$ (otherwise we drop from $S$ all slopes greater from $a^{\prime}$ without affecting the theorem). For every pair of two convex sets $d_{i}<d_{j}$ from $D$ we consider the set of directions for which there is a directed line meeting $d_{i}$ before $d_{j}$. These directions correspond to a closed arc $\left(d_{i}, d_{j}\right)$ on the unit circle which covers strictly less than a half-circle. The set of all linear functions corresponds to the half-circle of the unit circle contained in the $x$-positive half plane. Let $\left(d_{i}^{\prime}, d_{j}^{\prime}\right)$ be the intersection of this half-circle with the arc $\left(d_{i}, d_{j}\right)$. Since the latter arc covers strictly less than a half-circle, $\left(d_{i}^{\prime}, d_{j}^{\prime}\right)$ is a (continuous) arc as well. Thus projecting each $\left(d_{i}^{\prime}, d_{j}^{\prime}\right)$ stereographically from $(-1,0)$ to the $x=1$, we get a family of closed segments $\left(e_{i}, e_{j}\right)$ on a line that satisfy Theorem 2.10(ii) with $p=1$. The least common point corresponds to a direction $a^{\prime \prime} \in S$, in which every pair $d_{i}<d_{j}$ is met by a directed line consistently with the order. For every $d_{k} \in D$ we denote by $\operatorname{seg}[k]$ the interval resulted by the intersection of the $y$-axis with all the line transversals with slope $a^{\prime \prime}$ that $d_{k}$ admits. Since each two sets from $D$ admit a common transversal with slope $a^{\prime \prime}$, the intervals in $\left\{\operatorname{seg}[k] \mid d_{k} \in D\right\}$ are pairwise intersecting. Due to Helly's Theorem, 
there exists a point $b$ common to all the intervals. Let $b^{\prime \prime}$ be the minimal such $b$. In other words the line $y=a^{\prime \prime} x+b^{\prime \prime}$ is a line transversal for $D$.

If $a^{\prime \prime}<a^{\prime}$ then $\left(a^{\prime \prime}, b^{\prime \prime}\right)<_{L}\left(a^{\prime}, b^{\prime}\right)$ and we are done. Otherwise $\left(a^{\prime \prime}=a^{\prime}\right)$, we need to show that $b^{\prime \prime} \leq b^{\prime}$, i.e., $\forall k, \operatorname{seg}[k] \cap\left(-\infty, b^{\prime}\right] \neq \emptyset$. Since $a^{\prime \prime}=a^{\prime}$ there exists an interval of slopes $\left(d_{i^{\prime}}, d_{j^{\prime}}\right)$ whose minimal slope is $a^{\prime}$. Let us consider the interval of slopes of all the line transversals for $d_{k}, d_{i^{\prime}}, d_{j^{\prime}}$. The minimal slope in the interval is $a^{\prime}$. This implies together with the theorem's assumption about quadruple of objects, that there exists a common line transversal $y=a^{\prime} x+b$, for $d_{k}, d_{i^{\prime}}, d_{j^{\prime}}$ with $b \leq b^{\prime}$, so $\operatorname{seg}[k] \cap\left(-\infty, b^{\prime}\right] \neq \emptyset$ as needed. (Note that in this paragraph we use a weaker assumption that each triple of objects admits a line transversal $y=a x+b$ with $a \in S$ and $(a, b) \leq_{L}\left(a^{\prime}, b^{\prime}\right)$.)

Regarding the non-lexicographic discrete case, due to Observation $3.7 h^{*}$ is bounded from above by 4 . It is bounded from below by 4 as well due to Example 5.5.

The proof of the lexicographic continuous case is as follows. Observation 3.3 coupled with the standard version of the theorem (due to [23]) implies a lower bound of 3. It remains to show an upper bound of 3. Due to the theorem of [23] the arcs $\left(d_{i}, d_{j}\right)$ have a common point, so also do the segments $\left(e_{i}, e_{j}\right)$. Clearly the least such common point is the least point of a certain arc $\left(e_{i^{\prime}}, e_{j^{\prime}}\right)$. Let $a^{\prime \prime}$ be the direction corresponding to this point, in which every pair $d_{i}<d_{j}$ is met by a directed line consistently with the order. We conclude the proof for this case in the same way we did for the lexicographic discrete case.

\subsubsection{Theorem 2.18}

We start by proving the lexicographic discrete case. We can assume that $\forall a \in S$, $a \leq a^{\prime}$ (otherwise we drop from $S$ all slopes greater from $a^{\prime}$ without affecting the theorem). For every pair of two convex sets $d_{i}<d_{j}$ from $D$ we consider the set of directions for which there is a directed line meeting $d_{i}$ before $d_{j}$. These directions correspond to a closed arc $\left(d_{i}, d_{j}\right)$ on the unit circle which covers strictly less than a half-circle. Since every 4 members have a line transversal consistent with the order, this implies that the family of arcs intersect pairwise in a point from $\left(d_{i}, d_{j}\right)$. By the corresponding non-lexicographic Helly theorem $[22,23]$ there exists a directed line that meets the sets consistently with the order. Let $a_{0}$ be the direction of this line. Since each arc contains $a_{0}$ and is contained in a half-circle, none of the arcs contain the direction $-s_{0}$. Thus projecting stereographically from $-a_{0}$ to a line orthogonal to $a_{0}$, we get a family of closed segments on a line that satisfy Theorem 2.10(ii) with $p=1$. The least common point corresponds to a direction $a^{\prime \prime} \in S$, in which every pair $d_{i}<d_{j}$ is met by a directed line consistently with the order. For every $d_{k} \in D$ we denote by $\operatorname{seg}[k]$ the interval resulted by the intersection of the $y$-axis with all the line transversals with slope $a^{\prime \prime}$ that $d_{k}$ admits. Since each two sets from $D$ admit a common transversal with slope $a^{\prime \prime}$, the intervals in $\left\{\operatorname{seg}[k] \mid d_{k} \in D\right\}$ are pairwise intersecting. Due to Helly's Theorem, there exists a point $b$ common to all the intervals. Let $b^{\prime \prime}$ be the minimal such $b$. In other words the line $y=a^{\prime \prime} x+b^{\prime \prime}$ is a line transversal for $D$. Since every two sets are stabbed by some translate of this directed line consistently with the order, the disjointness of the sets implies that the line $y=a^{\prime \prime} x+b^{\prime \prime}$ stabs the sets consistently with the order as well. 
If $a^{\prime \prime}<a^{\prime}$ then $\left(a^{\prime \prime}, b^{\prime \prime}\right)<_{L}\left(a^{\prime}, b^{\prime}\right)$ and we are done. Otherwise $\left(a^{\prime \prime}=a^{\prime}\right)$, we need to show that $b^{\prime \prime} \leq b^{\prime}$, i.e., $\forall k, \operatorname{seg}[k] \cap\left(-\infty, b^{\prime}\right] \neq \emptyset$. Since $a^{\prime \prime}=a^{\prime}$ there exists an interval of slopes $\left(d_{i^{\prime}}, d_{j^{\prime}}\right)$ whose minimal slope is $a^{\prime}$. Let us consider the interval of slopes of all the line transversals for $d_{k}, d_{i^{\prime}}, d_{j^{\prime}}$. The minimal slope in the interval is $a^{\prime}$. This implies together with the theorem's assumption about quadruple of objects, that there exists a common line transversal $y=a^{\prime} x+b$, for $d_{k}, d_{i^{\prime}}, d_{j^{\prime}}$ with $b \leq b^{\prime}$, so $\operatorname{seg}[k] \cap\left(-\infty, b^{\prime}\right] \neq \emptyset$ as needed.

Regarding the non-lexicographic discrete case, due to Observation $3.7 h^{*}$ is bounded from above by 4 . It is bounded from below by 4 as well due to Example 5.5.

The proof of the lexicographic continuous version is similar. We use Theorem 2.7(ii) instead of Theorem 2.10(ii), and note that the Helly number 4 is tight due to Observation 3.3 and the non-lexicographic continuous version of this theorem which has Helly number 4, i.e., the theorem which was independently proved by Tverberg [22] and Wenger [23].

\subsubsection{Theorem 2.15}

In the following two subsections we use a well known (see for example [7]) geometric duality transformation.

Definition 5.1 Let $\mathcal{D}$ be a transformation which maps every point $(a, b)$ in the plane into the non vertical line $y=a x-b$ and vice versa, that is, it maps a non vertical line $l$ to the point $\mathcal{D}(l)$ such that $\mathcal{D}(\mathcal{D}(l))=l$. Let $h \subset \mathbb{R}^{2}$ be a set of points. We extend the definition of $\mathcal{D}$ to sets of points in the natural way $\mathcal{D}(h)=\{\mathcal{D}(p) \mid p \in h\}$.

Observation 5.2 For every $a, b \in \mathbb{R}$ and set $h \in \mathbb{R}^{2}$, the line $y=a x+b$ intersects the set $h$ if and only if $(a, b) \in \mathcal{D}(h)$.

We define in the dual space the dual lexicographic order $\leq_{L^{\prime}}$ as $(a, b) \leq_{L^{\prime}}\left(a^{\prime}, b^{\prime}\right)$ if and only if either $a<a^{\prime}$ or $a=a^{\prime}$ and $b \geq b^{\prime}$, for every reals $a, a^{\prime}, b$ and $b^{\prime}$. In this way due to Observation 5.2 we get that given $\left(a^{\prime}, b^{\prime}\right) \in \mathbb{R}^{2}$ and a set $h \in \mathbb{R}^{2}$, there exists a line $y=a x+b$ with $(a, b) \leq_{L}\left(a^{\prime}, b^{\prime}\right)$ that intersects $h$, if and only if there exists a point $\left(a^{\prime \prime}, b^{\prime \prime}\right)$ in the dual space such that $\left(a^{\prime \prime}, b^{\prime \prime}\right) \leq_{L^{\prime}}\left(a^{\prime}, b^{\prime}\right)$.

Observation $5.3 \mathcal{D}$ transforms every vertical interval $\left[(a, b),\left(a, b^{\prime}\right)\right]$ into a strip with slope $a$, which intersects the $y$-axis in the interval $\left[\left(0,-b^{\prime}\right),(0,-b)\right]$ and transforms the set of all lines with slope a into the vertical line $x=a$.

Observation $5.4 \mathcal{D}$ transforms every axis-parallel rectangle $h$ into an unbounded non-convex polygon $\mathcal{D}(h)$, which is the complement of the union of two disjoint unbounded convex polygons. The intersection of $\mathcal{D}(h)$ with either one of the $x$ nonpositive or the $x$ non-negative halfplanes yields a convex unbounded polygon (see Fig. 6).

Case (i): We note that since we deal with parallel line segments, the order in which a line meets any subset of the line segments is uniquely defined. Therefore, the upper bounds for this case are due to Theorem 2.17. We choose to give a direct proof for the 
Fig. 6 Two rectangles and their corresponding line transversals
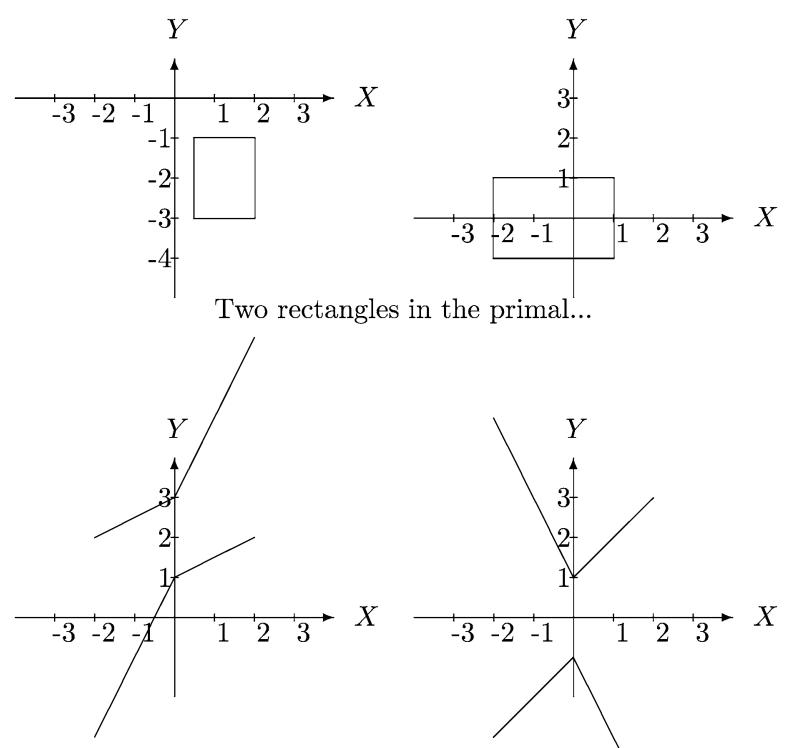

$X$

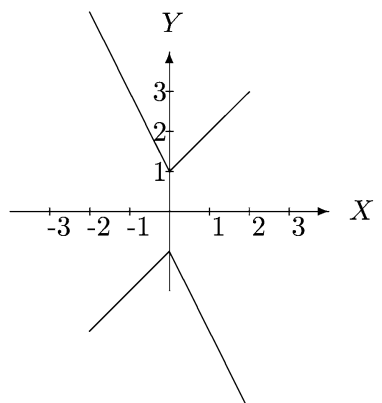

$\ldots$ and their corresponding line transversals in the dual

lexicographic continuous case since it is short and gives intuition to how we prove Case (ii).

In order to prove $h_{L}=3$ we use the duality transformation $\mathcal{D}$ defined above, and consider the corresponding dual problem. Due to Observation 5.3, each vertical line segment in the primal is transformed to a strip in the dual. Since the resulting strips are convex sets, we get from the Lexicographic Helly's Theorem (Theorem 2.1) that if every 3 strips intersect in a point lexicographically at most $\left(a^{\prime}, b^{\prime}\right)$, then all strips do as well. Every point in this intersection equals in the primal problem to a line transversal $y=a x+b$ with $(a, b) \leq_{L}\left(a^{\prime}, b^{\prime}\right)$ which intersects $H$. Note that this Helly number cannot be reduced to two (consider for instance any 3 non-collinear points in the plane).

Regarding the lexicographic discrete case, applying Theorem 2.17, we get an upper bound of 4 . By choosing $a^{\prime}$ arbitrary large, say $a^{\prime}=10$, and any real $b^{\prime}$ it is clear from the following example that the Helly number cannot be reduced to 3 :

Example 5.5 Let $D=\{a, b, c, d\}$ consist of the vertical 2-units intervals $a=$ $[(-1.5,1) ;(-1.5,3)], b=[(-1,2) ;(-1,4)], c=[(1,2) ;(1,4)]$ and $d=[(1.5,3)$; $(1.5,5)]$ (see left side of Fig. 7) and let $S=\left\{s_{1}=-0.1, s_{2}=1.1\right\}$ be the set of line directions (each direction is represented by a real number). The set of line transversals for each interval is a strip in the dual (see right side of Fig. 7). The feasible region of line transversals is the intersection of the 4 strips $\mathcal{D}(a), \mathcal{D}(b), \mathcal{D}(c), \mathcal{D}(D)$, i.e., the quadrangle whose corners are the white points in the figure. The set of line transversals with directions in $S$ are the two thick vertical lines $\mathcal{D}\left(s_{1}\right), \mathcal{D}\left(s_{2}\right)$. We note that the quadrangle does not intersect either of the lines $\mathcal{D}\left(s_{1}\right), \mathcal{D}\left(s_{2}\right)$, while the intersection of each three strips intersects one of the two vertical lines. 


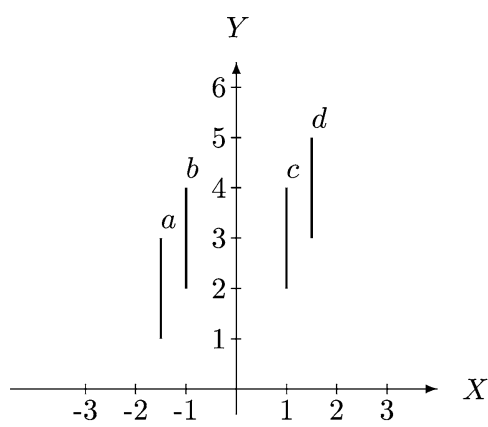

The set of the intervals in the primal

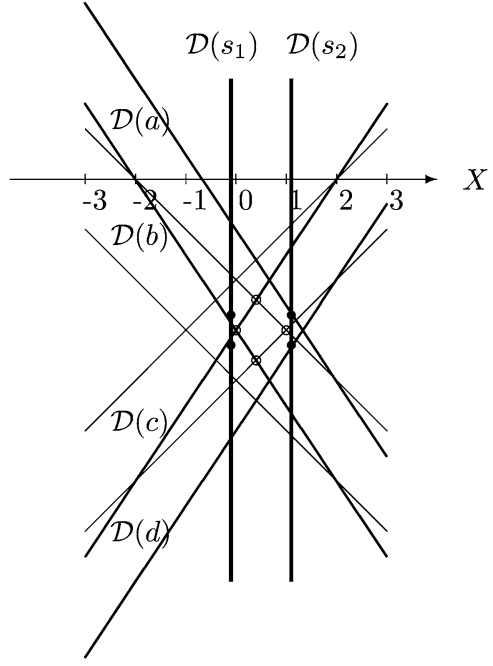

The set of feasible line transversals in the dual

Fig. 7 An instance of Theorem 2.15(i)

As for the non-lexicographic discrete case, due to Observation 3.7 the lexicographic discrete case gives an upper bound of 4 . This number cannot be reduced due to Example 5.5.

Case (ii): We will use the duality transformation $\mathcal{D}$ from Definition 5.1 . We start by proving that the Helly number of the lexicographic continuous version is 5 . Let $\omega: 2^{H} \rightarrow \mathbb{R}^{2} \cup\{\infty\}$ be a function such that for every $H^{\prime} \subseteq H, \omega\left(H^{\prime}\right)$ is the lexicographically smallest point in $\bigcap_{h \in H^{\prime}} \mathcal{D}\left(h^{\prime}\right)$, if such intersection is not empty, and $\infty$ otherwise. The definition of $\omega$ together with Observation 5.2 implies that for every $a^{\prime}, b^{\prime} \in \mathbb{R}$ and $H^{\prime} \subseteq H$, the rectangles in $H^{\prime}$ are met by a common line $y=a x+b$ with $(a, b) \leq_{L}\left(a^{\prime}, b^{\prime}\right)$ if and only if $\omega\left(H^{\prime}\right) \leq_{L^{\prime}}\left(a^{\prime}, b^{\prime}\right)$. Moreover, since set intersection is monotone (i.e., $A \cap B \subseteq A, B),(H, \omega)$ is an abstract problem which meets the Monotonicity Condition. Hence, due to Theorem 4.5, it is sufficient to show that for every $H^{\prime} \subseteq H$, there exists a solution defining set $H^{\prime \prime}$ of cardinality at most 5 .

If there is no line transversal for $H^{\prime}$, by Theorem 2.14(iii) there are at most 5 translates in $H$ which do not admit a common line transversal. We set $H^{\prime \prime}$ to consist of these translates.

Otherwise, $H^{\prime}$ admits a line transversal. Due to Observation 5.4 the intersection of $\bigcap_{h \in H^{\prime}} \mathcal{D}(h)$ with the $x$ non-negative halfplane (the $x$ non-positive halfplane) is a (possibly empty) convex polygon $P_{+}\left(P_{-}\right)$, respectively. If $P_{-}$is not empty, there exist at most two translates $h_{1}, h_{2} \in H^{\prime}$ which determine the lexicographically smallest point in $P_{-}$(i.e., lexicographic min $P_{-}=$lexicographic $\left.\min \left(\mathcal{D}\left(h_{1}\right) \cap \mathcal{D}\left(h_{2}\right)\right)\right)$. We set $H^{\prime \prime}=\left\{h_{1}, h_{2}\right\}$.

Otherwise ( $P_{-}$is empty and $P_{+}$is not empty), due to Helly's Theorem there exists a set $H^{\prime \prime \prime} \subseteq H^{\prime}$ of cardinality at most 3 such that $\left(\mathbb{R}^{-} \times \mathbb{R}\right) \cap\left(\bigcap_{h \in H^{\prime \prime \prime}} \mathcal{D}(h)\right)=\emptyset$. Moreover, since $P_{+}$is not empty there exist at most two translates $h_{1}, h_{2} \in H^{\prime}$ which determine the lexicographically smallest point in $P_{+}$(i.e., lexicographic $\min P_{+}=$ 
lexicographic $\left.\min \left(\left(\mathbb{R}^{+} \times \mathbb{R}\right) \cap \mathcal{D}\left(h_{1}\right) \cap \mathcal{D}\left(h_{2}\right)\right)\right)$. In this case we set $H^{\prime \prime}=H^{\prime \prime \prime} \cup$ $\left\{h_{1}, h_{2}\right\}$.

In all cases, $H^{\prime \prime}$ is a solution defining set for $H^{\prime}$ with cardinality at most 5 . The Helly number is bounded from below by 5 due to Observation 3.7 and the fact that the Helly number of the corresponding non-lexicographic Helly theorem, Theorem 2.14(iii), is 5.

We now consider the lexicographic discrete version. Let $\omega: 2^{D} \times 2^{S} \rightarrow \mathbb{R}^{2} \cup\{\infty\}$ be a function such that for every $\left(D^{\prime}, S^{\prime}\right) \in 2^{D} \times 2^{S}, \omega\left(D^{\prime}, S^{\prime}\right)$ is the lexicographically smallest point in $\bigcap_{d^{\prime} \in D^{\prime}} \mathcal{D}\left(d^{\prime}\right)$ with $x$-coordinate in $S^{\prime}$, if such intersection is not empty, and $\infty$ otherwise. The definition of $\omega$ together with Observation 5.2 implies that for every $a^{\prime}, b^{\prime} \in \mathbb{R}$ and $\left(D^{\prime}, S^{\prime}\right) \in 2^{D} \times 2^{S}$, the rectangles in $D^{\prime}$ are met by a common line $y=a x+b$ with $(a, b) \leq_{L}\left(a^{\prime}, b^{\prime}\right)$ and $a \in S^{\prime}$ if and only if $\omega\left(D^{\prime}, S^{\prime}\right) \leq_{L^{\prime}}\left(a^{\prime}, b^{\prime}\right)$. Moreover, since set intersection is monotone (i.e., $A \cap B \subseteq A, B),(D, S, \omega)$ is a discrete abstract problem (see Definition 4.7), which meets the Monotonicity of Demand Condition. Hence, due to Theorem 4.10, it is sufficient to show that for every $\left(D^{\prime}, S^{\prime}\right) \in 2^{D} \times 2^{S}$, there exists a solution defining set $\left(D^{\prime \prime}, S^{\prime}\right)$ with $\left|D^{\prime \prime}\right| \leq 7$.

We first consider the case where $D^{\prime}$ admits a line transversal. Let $P_{+}\left(P_{-}\right)$denote the intersection of $\bigcap_{d^{\prime} \in D^{\prime}} \mathcal{D}\left(d^{\prime}\right)$ with the $x$ non-negative (the $x$ non-positive) halfplane, respectively. Due to Observation 5.4, $P_{+}$and $P_{-}$are (possibly empty) convex polygons.

For $X=P_{-}, P_{+}$, let $d^{l}(X), d_{l}(X) \in D^{\prime}$ be rectangles such that the leftmost intersection point of $\mathcal{D}\left(d^{l}\right), \mathcal{D}\left(d_{l}\right)$ equals the leftmost point in $X$. Similarly, let $d^{r}(X), d_{r}(X) \in D$ be such that the rightmost intersection point of $\mathcal{D}\left(d^{r}\right), \mathcal{D}\left(d_{r}\right)$ equals the rightmost point in $X$. In this way at most 8 rectangles determine the leftmost and rightmost extreme points in $P_{-}$and $P_{+}$.

Observations 5.2 and 5.3, and the definition of $P_{+}$and $P_{-}$imply that $D^{\prime}$ admits a line transversal with direction in $S^{\prime}$ if and only if the intersection between $P_{+} \cup P_{-}$and $\mathcal{D}\left(S^{\prime}\right)$ is not empty. By making a more careful analysis, which takes into consideration the fact that the objects in $D$ are pairwise disjoint translates of a rectangle, we will show that the leftmost and rightmost points in $P_{+}$and $P_{-}$are determined by at most 7 polygons. I.e., we can choose $\left\{d^{l}\left(P_{-}\right), d_{l}\left(P_{-}\right), d^{r}\left(P_{-}\right), d_{r}\left(P_{-}\right), d^{l}\left(P_{+}\right), d_{l}\left(P_{+}\right), d^{r}\left(P_{+}\right), d_{r}\left(P_{+}\right)\right\}$such that the set is of cardinality at most 7 .

The projection of $P_{+}$on the $x$-axis results in an interval $\left[s l^{\min }, s l^{\max }\right]$ of nonnegative slopes with which there exists a line transversal for $D^{\prime}$. Similarly, the projection of $P_{-}$on the $x$-axis results an interval $\left[s l_{\min }, s l_{\max }\right]$ of non-positive slopes with which there exists a line transversal for $D^{\prime}$. We call $\left[s l_{\min }, s l_{\max }\right] \cup\left[s l^{\min }, s l^{\max }\right]$ the slope range corresponding to $D^{\prime}$. The 2 rectangles $d^{l}\left(P_{-}\right), d_{l}\left(P_{-}\right)$determine $s l_{\min }$, the minimal non-positive slope of a line transversal for $D^{\prime}$. Similarly, $d^{r}\left(P_{+}\right), d_{r}\left(P_{+}\right)$ determine $s l^{\max }$, the maximal non-negative slope of a line transversal for $D^{\prime}$. For example, let $D_{1}$ be the set consisting of the 4 pairwise disjoint translates of a square as drawn in Fig. 8. Rectangles 1 and 2 determine the maximal non-negative slope of a line transversal, and rectangles 3 and 4 determine the minimal non-positive slope of a line transversal. In this example the range of non-positive slopes of line transversals for $D_{1}$ is $\left[s l_{\min }, s l_{\max }\right]=[-0.2,0]$ and the range of non-negative slopes is $\left[s l^{\mathrm{min}}, s l^{\mathrm{max}}\right]=[0,0.2]$. Any subset of $D_{1}$ containing only 3 rectangles has a slope 


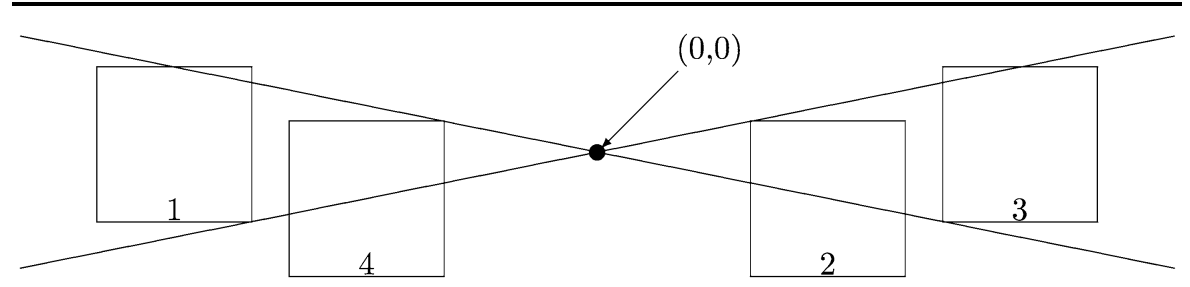

Fig. 84 squares admitting line transversals with slopes between $-\frac{1}{5}$ to $\frac{1}{5}$

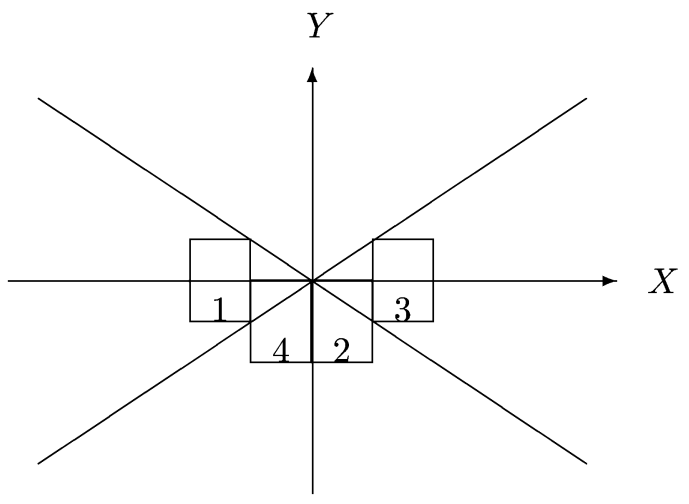

(a)

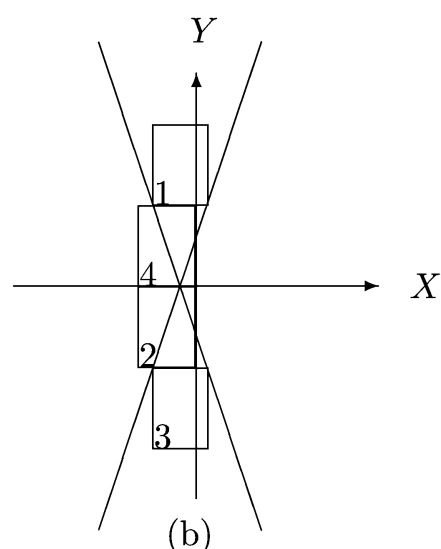

(b)

Fig. 98 rectangles and their corresponding line transversals slope ranges

range strictly containing the slope range corresponding to $D_{1}$. We note that moving the squares in Fig. 8 towards the origin, may cause their slope range to strictly contain the slope range corresponding to $D_{1}$. Let $D_{2}$ be the set consisting of the 4 squares in a figure such as Fig. 8 rotated by $\frac{\Pi}{2}$ radians relatively to the origin. The slope range corresponding to $D_{2}$ is $\left[s l_{\min }, s l_{\max }\right]=[-\infty,-5]$ and $\left[s l^{\min }, s l^{\max }\right]=[5, \infty]$. Any subset of $D_{2}$ containing only 3 rectangles has a slope range strictly containing the slope range corresponding to $D_{2}$. Again, we note that moving the squares towards the origin may result in a slope range strictly containing the original one. We note that $D_{1} \cup D_{2}$ does not admit a line transversal, since the intersection of the slope ranges corresponding to $D_{1}$ and $D_{2}$ is empty. We now show that it is not possible to move the squares in $D_{1} \cup D_{2}$ towards the origin, while keeping the squares pairwise disjoint, such that the slope range corresponding to the 8 translated squares is not empty and is different from the slope range corresponding to any subset of 7 squares. We will show that the above holds even if we take translates of a rectangle instead of a square.

Let $y$ denote the height of the rectangle $O$ and let $x$ denote its width. In Fig. 9a the 4 rectangles are moved as much as possible towards the origin. Let $D_{1}^{\prime}$ be the set consisting of these 4 rectangles, and let $\alpha$ be the maximal angle of a line transversal for $D_{1}^{\prime}$. We get that $\alpha=\arctan \frac{y}{2 x}$. Similarly, in Fig. $9 \mathrm{~b}$ the 4 rectangles are moved as much as possible towards the origin. Let $D_{2}^{\prime}$ be the set consisting of these 4 rectangles, and let $\beta$ be the angle between the $y$-axis and the line transversal for $D_{2}^{\prime}$ with the 
minimal positive slope. We get that $\beta=\arctan \frac{x}{2 y}$. (We note that a rectangle in $D_{1}^{\prime}$ may have a non-empty intersection with a rectangle in $D_{2}^{\prime}$.) In order to get that $D_{1}^{\prime} \cup$ $D_{2}^{\prime}$ admits a line transversal, we must have $\alpha+\beta \geq \frac{\Pi}{2}$. To conclude our proof it is therefore sufficient to show that $\alpha+\beta<\frac{\Pi}{2}$.

Let $z=\frac{y}{x}$ be the proportion of the rectangle $O(z=1$ if and only if the rectangle is a square). Then $\alpha=\arctan \frac{z}{2}$ and $\beta=\arctan \frac{1}{2 z}$. Let

$$
f(z)=\alpha+\beta=\arctan \frac{z}{2}+\arctan \frac{1}{2 z} .
$$

Then

$$
f^{\prime}(z)=\frac{2}{4+z^{2}}-\frac{2}{4 z^{2}+1},
$$

which equals 0 whenever $z^{2}=1$.

$$
f^{\prime \prime}(z)=-\frac{4 z}{\left(4+z^{2}\right)^{2}}+\frac{16 z}{\left(4 z^{2}+1\right)^{2}} .
$$

We get $f^{\prime \prime}(1)>0$ so $f(z)$ attains its minimum in $z=1\left(f(1)=2 \arctan \frac{1}{2}<\frac{\Pi}{2}\right)$. We note that for any other positive finite value for $z$ we still get $\alpha+\beta=f(z)<\frac{\Pi}{2}$ as needed.

We now determine a solution defining set for $D^{\prime}$. If $P_{+} \cup P_{-}$does not intersect any of the vertical lines in $\mathcal{D}\left(S^{\prime}\right)$ we set $D^{\prime \prime}=\left\{d^{l}\left(P_{-}\right), d_{l}\left(P_{-}\right), d^{r}\left(P_{-}\right), d_{r}\left(P_{-}\right), d^{l}\left(P_{+}\right)\right.$, $\left.d_{l}\left(P_{+}\right), d^{r}\left(P_{+}\right), d_{r}\left(P_{+}\right)\right\}$.

Otherwise, let $x=a$ the vertical line in $\mathcal{D}\left(S^{\prime}\right)$ with the minimal $a$ which $P_{+} \cup P_{-}$ intersects. We let $d^{*} \in D^{\prime}$ be a rectangle such that the lowest (w.r.t. the $y$-axis) intersection point $\left(a^{\prime}, y^{\prime}\right)$, of the polygon $\mathcal{D}\left(d^{*}\right)$ with the line $x=a$, has the highest $y^{\prime}$. If $P_{-}$intersects the line $x=a$ we set $D^{\prime \prime}=\left\{d^{l}\left(P_{-}\right), d_{l}\left(P_{-}\right), d^{*}\right\}$. Otherwise $P_{+}$intersects the line $x=a$ and we set $D^{\prime \prime}=\left\{d^{l}\left(P_{-}\right), d_{l}\left(P_{-}\right), d^{r}\left(P_{-}\right), d_{r}\left(P_{-}\right), d^{l}\left(P_{+}\right)\right.$, $\left.d_{l}\left(P_{+}\right), d^{*}\right\}$.

If $D^{\prime}$ does not admit a line transversal, then due to Theorem 2.14(v) there are at most 5 translates in $D^{\prime}$ which do not admit a line transversal. We set $D^{\prime \prime}$ to consist of these rectangles.

In all cases, $\left(D^{\prime \prime}, S^{\prime}\right)$ is a solution defining set for $\left(D^{\prime}, S^{\prime}\right)$ with cardinality at most 7. Example 5.6 shows that the Helly number is bounded from below by 7 , even for the spacial case of squares.

Example 5.6 Let $D=\{a, b, c, d, e, f, g\}$ consist of the 4-units length open squares such that $a$ is centered at $(2,2) ; b$ is centered at $\left(-2 \frac{1}{4}, 0\right) ; c$ is centered at $\left(-2 \frac{1}{9}, 4\right)$; $d$ is centered at $\left(\frac{4}{5}, 8\right), e$ is centered at $\left(-1 \frac{1}{2},-4\right) ; f$ is centered at $(-1,12)$; and $g$ is centered at $\left(\frac{5}{7},-8\right)$. Let $a^{\prime}=b^{\prime}=100$.

We observe that $a$ and $b$ determine $s l^{\max }=8, a$ and $c$ determine $s l_{\min }=-18$, $d$ and $e$ determine $s l^{\min }=4 \frac{12}{17}$, and $f$ and $g$ determine $s l_{\max }=-7$. Hence the corresponding slope range is $[-18,-7] \cup\left[4 \frac{12}{17}, 8\right]$. Let $S=\left\{-18-\epsilon,-7+\epsilon, 4 \frac{12}{17}-\epsilon\right.$, $8+\epsilon\}$ (all of these slopes are smaller than $a^{\prime}$ ). Since $D$ is finite it is possible to choose 
$\epsilon>0$ small enough such that $D$ does not have a line transversal with direction in $S$ but any of its proper subsets does.

(We note that there exists $\delta>0$ small enough such that we can modify this example to closed squares of length $4-\delta$, so the corresponding slope range will not change significantly, and consequently the set of slope directions will be similar to $S$.)

Last, we consider the non-lexicographic discrete version. Observation 3.7 coupled with the fact that the corresponding lexicographic discrete Helly number is 7, implies an upper bound of 7. Example 5.6 gives us a lower bound of 7 .

\subsubsection{Theorem 2.13}

We start by proving the lexicographic continuous Helly number. We consider the corresponding dual problem. Due to Observation 5.4, each rectangle $d \in D$ in the primal is transformed into a polygon $\mathcal{D}(d)$ such that its intersection with the $x$ nonnegative halfplane is a convex polygon $p(d)$. Due to Observation 5.2, $D$ admits a line transversal $y=a x+b$ with a non-negative slope $a$ and $(a, b) \leq_{L}\left(a^{\prime}, b^{\prime}\right)$ if and only if the convex polygon $\bigcap_{d \in D} p(d)$ contains a point which is not lexicographically greater than $\left(a^{\prime}, b^{\prime}\right)$. Due to the Lexicographic Helly's Theorem (Theorem 2.1) on the convex polygons $p(d), \forall d \in D$ we get an upper bound of 3 . Observation 3.3 and the corresponding non-lexicographic continuous Helly number (The theorem of Hadwiger and Debrunner [15]) give us a lower bound of 3, as needed.

We now prove a lex-discrete Helly number by using Theorem 4.10. We will use the duality transformation $\mathcal{D}$ from Definition 5.1. Let $\omega: 2^{D} \times 2^{S} \rightarrow \mathbb{R}^{2} \cup\{\infty\}$ be a function such that for every $\left(D^{\prime}, S^{\prime}\right) \in 2^{D} \times 2^{S}, \omega\left(D^{\prime}, S^{\prime}\right)$ is the lexicographically smallest point in $\bigcap_{d^{\prime} \in D^{\prime}} \mathcal{D}\left(d^{\prime}\right)$ with $x$-coordinate in $S^{\prime}$, if such intersection is not empty, and $\infty$ otherwise. The definition of $\omega$ together with Observation 5.2 implies that for every $a^{\prime}, b^{\prime} \in \mathbb{R}$ and $\left(D^{\prime}, S^{\prime}\right) \in 2^{D} \times 2^{S}$, the rectangles in $D^{\prime}$ are met by a common line $y=a x+b$ with $(a, b) \leq_{L}\left(a^{\prime}, b^{\prime}\right)$ and $a^{\prime} \in S^{\prime}$ if and only if $\omega\left(D^{\prime}, S^{\prime}\right) \leq_{L^{\prime}}\left(a^{\prime}, b^{\prime}\right)$. Moreover, since set intersection is monotone (i.e., $A \cap B \subseteq A, B),(D, S, \omega)$ is a discrete abstract problem (see Definition 4.7), which meets the Monotonicity of Demand Condition. Hence, due to Theorem 4.10, it is sufficient to show that for every $\left(D^{\prime}, S^{\prime}\right) \in 2^{D} \times 2^{S}$, there exists a solution defining set $\left(D^{\prime \prime}, S^{\prime}\right)$ with $\left.\left|D^{\prime \prime}\right| \leq 4\right)$.

If $D^{\prime}$ does not admit a line transversal, by the corresponding non-lexicographic Helly theorem by [15] there are 3 rectangles in $D^{\prime}$ which do not admit a common line transversal. We let $D^{\prime \prime}$ be the set consisting of these rectangles.

Otherwise we let $P_{+}, d^{l}\left(P_{+}\right), d_{l}\left(P_{+}\right), d^{r}\left(P_{+}\right)$and $d_{r}\left(P_{+}\right)$be as defined in the proof of Theorem 2.15(ii). If $P_{+}$does not intersect any of the vertical lines in $\mathcal{D}\left(S^{\prime}\right)$ we set $D^{\prime \prime}=\left\{d^{l}\left(P_{+}\right), d_{l}\left(P_{+}\right), d^{r}\left(P_{+}\right), d_{r}\left(P_{+}\right)\right\}$.

If $P_{+}$does intersect $\mathcal{D}\left(S^{\prime}\right)$, we let $x=a$ be the vertical line in $\mathcal{D}(S)$ with the minimal $a$ which $P_{+}$intersects. We let $d^{*} \in D^{\prime}$ be such that the lowest (w.r.t. the $y$-axis) intersection point $\left(a^{\prime}, y^{\prime}\right)$, of the polygon $\mathcal{D}\left(d^{*}\right)$ with the line $x=a$, has the highest $y^{\prime}$. We set $D^{\prime \prime}=\left\{d^{l}\left(P_{+}\right), d_{l}\left(P_{+}\right), d^{*}\right\}$.

In all cases, $\left(D^{\prime \prime}, S^{\prime}\right)$ is a solution defining set for $\left(D^{\prime}, S^{\prime}\right)$ with cardinality at most 4. The Helly number cannot be reduced to 3 due to Example 5.5.

As for the non-lexicographic discrete Helly number, Example 5.5 gives us a lower bound of 4 . We get an upper bound of 4 due to the lex-discrete Helly number and Observation 3.7. 
We conclude this section by observing that in the proof of the above theorem we also showed the following observation:

Observation 5.7 Let $D$ be a family of axis-parallel rectangles in the plane and for every $d \in D$, let $\mathcal{L}(d)$ be the set of line transversals which $d$ admits in the dual (see Definition 5.1). Let $P(D)=\bigcap_{d \in D} \mathcal{L}(d)$ be the set of line transversals which $D$ admits. The intersection of $P(D)$ with either the $x$ non-negative or $x$ non-positive halfplanes is a convex polygon. The slopes of line transversals with non-negative (nonpositive) slopes for $D$ generate an interval $\left[s l^{\min }, s l^{\max }\right]\left(\left[s l_{\min }, s l_{\max }\right]\right)$ resulted by the projection of $P(D)$ on the non-negative (non-positive) part of the $x$-axis, respectively. Each of the 4 endpoints of these two intervals is determined by two rectangles.

If in the last case considered in the proof of the theorem above, we set $D^{\prime \prime}$ to be $D^{\prime \prime}=\left\{d^{l}\left(P_{+}\right), d_{l}\left(P_{+}\right), d^{r}\left(P_{+}\right), d_{r}\left(P_{+}\right), d^{*}\right\}$ (instead of $\left.\left\{d^{l}\left(P_{+}\right), d_{l}\left(P_{+}\right), d^{*}\right\}\right)$, we get the following somewhat stronger Helly theorem:

Theorem 5.8 Let $D$ be a family of rectangles in the plane with edges parallel to the axes and let $S$ be a set of non-negative reals (line directions). For every pair of reals $a^{\prime} \geq 0$ and $b^{\prime}$, and pair of non-negative reals $s l_{\min } \leq s l_{\max }$, if every subfamily of at most 5 rectangles admits a line transversal $y=a x+b$ with $a \in S$ and $s l_{\min } \leq a \leq$ $s l_{\max }$ and $(a, b) \leq_{L}\left(a^{\prime}, b^{\prime}\right)$ then $D$ does as well.

\section{Concluding Remarks}

In this paper we introduce the notion of discrete Helly theorems and lexicographic Helly theorems. We obtain numerous new such Helly theorems. We prove some of these results via "abstract problems" and "solution defining sets", which we formally define in Sect. 4. We also study the relations between the different types of Helly theorems.

We strongly believe that many more discrete and lexicographic Helly theorems exist. For instance, regarding a discrete version for Tverberg's theorem [21],

Conjecture Let $H$ be a set of pairwise disjoint translates of a compact convex set $O$ in the plane, and let $S$ be a set of reals (line directions). There exists a finite number $k$ such that if every subset of at most $k$ translates from $H$ is met by a common line $y=a x+b$ with slope $a \in S$, then $H$ does as well.

Tverberg's theorem [21] coupled with Observation 3.8 implies $k \geq 7$. We believe that the minimal such number $k$ is 7 .

Acknowledgements The author would like to thank Branko Grünbaum for inspiring discussions and an anonymous referee for many useful comments which greatly improved the presentation of this paper.

\section{References}

1. Amenta, N.: Helly-type theorems and generalized linear programming. Discrete Comput. Geom. 12, 241-261 (1994) 
2. Danzer, L.: Über ein problem aus der combinatorischen geometrie. Arch. Math. (Basel) 8, 344-351 (1957)

3. Danzer, L., Grünbaum, B.: Intersection properties of boxes in $\mathbb{R}^{d}$. Combinatorica 2, 237-246 (1982)

4. Danzer, L., Grünbaum, B., Klee, V.: Helly's theorem and its relatives. In: Proceedings of the Symposium on Pure Mathematics (Convexity), vol. 7, pp. 101-180. American Mathematical Society, Providence (1963)

5. Doignon, J.P.: Convexity in cristallographical lattices. J. Geom. 3, 71-85 (1973)

6. Eckhoff, J.: Helly, radon and Carathodory type theorems. In: Gruber, P.M., Willis, J.M. (eds.) Handbook of Convex Geometry. Elsevier, Amsterdam (1993)

7. Edelsbrunner, H.: Algorithms in Combinatorial Geometry. Springer, New York (1987)

8. Goodman, J.E., Pollack, R., Wenger, R.: Geometric transversal theory. In: Pach, J. (ed.) New Trends in Discrete and Computational Geometry. Springer, Berlin (1993)

9. Grünbaum, B.: On a theorem of L.A. Santaló. Pac. J. Math. 5, 351-359 (1955)

10. Grünbaum, B.: On common transversals. Arch. Math. (Basel) 9, 465-469 (1958)

11. Hadwiger, H.: Über eibereiche mit gemeinsamer treffgeraden. Port. Math. 16, 23-29 (1957)

12. Halman, N.: Discrete and lexicographic Helly theorems and their relations to LP-type problems. Ph.D. Dissertation, Tel Aviv University, 2004. Available online at http://www.math.tau.ac.il/phd/ dissertations/Halman_Nir.ps

13. Halman, N.: On the power of discrete and of lexicographic Helly theorems. In: Proceedings of the Forty Fifth Annual Symposium on Foundations of Computer Science, pp. 463-472 (2004)

14. Halman, N.: On the power of discrete and of lexicographic Helly theorems. SIAM J. Comput., to appear

15. Hadwiger, H., Debrunner, H.: Ausgewählte einzelprobleme der kombinatorischen geometrie in der ebene. Enseingn. Math. 1, 56-89 (1955)

16. Hadwiger, H., Debrunner, H., Klee, V.: Combinatorial Geometry in the Plane. Rinhart and Winston, New York (1964)

17. Helly, E.: Über mengen konvexer mit gemeinschaftlichen punkten. Jber. Dtsch. Math. Ver. 32, 175176 (1923)

18. Klee, V.L., Jr: Common secants for plane convex sets. Proc. Am. Math. Soc. 5, 639-641 (1954)

19. Matoušek, J.: Lectures on Discrete Geometry. Springer, New York (2002)

20. Santaló, L.: Un teorema sóbre conjuntos de paralelepipedos de aristas paralelas. Publ. Inst. Mat. Univ. Nac. Litoral 2, 49-60 (1940)

21. Tverberg, H.: Proof of Grünbaum's conjecture on common transversals for translates. Discrete Comput. Geom. 4, 191-203 (1989)

22. Tverberg, H.: On geometric permutations and the Katchalski-Lewis conjecture on partial transversals for translates. In: Discrete Computational Geometry: Papers from the DIMACS Special Year. American Mathematical Society, Providence (1991)

23. Wenger, R.: A generalization of Hadwiger's theorem to intersecting sets. Discrete Comput. Geom. 5, 383-388 (1990) 